\title{
Les Vite de 1672 de Bellori : hypothèses de reconstitution du programme iconographique et théorique
}

\author{
Jean-François Lhote
}

Volume 14, numéro 1-2, 1987

URI : https://id.erudit.org/iderudit/1073453ar

DOI : https://doi.org/10.7202/1073453ar

\section{Aller au sommaire du numéro}

\section{Éditeur(s)}

UAAC-AAUC (University Art Association of Canada | Association d'art des universités du Canada)

\section{ISSN}

0315-9906 (imprimé)

1918-4778 (numérique)

Découvrir la revue

Citer cet article

Lhote, J.-F. (1987). Les Vite de 1672 de Bellori : hypothèses de reconstitution du programme iconographique et théorique. RACAR : Revue d'art canadienne / Canadian Art Review, 14(1-2), 75-89. https://doi.org/10.7202/1073453ar

\section{Résumé de l'article}

This article presents the evidence for a tentative reconstruction of the theoretical and iconographic programme of Bellori's Vite. The author seeks to show that Bellori's biographies are arranged according to a programmatic scheme probably conceived in the late 1630 s or early 1640 s, during the biographer's youth, and that this programme is inspired by Lomazzo's Temple of Painting and the theory of Governors.

Published in 1672, the Vite present the lives of twelve artists, following - up to a point-the chronological order of their deaths, as was the rule in that literary genre. However, three of them (Annibale Carracci, Caravaggio, and Domenichino) are out of chronological order, and at the same time divide the whole group into three equal parts. This anomaly is explained by a poem published in 1642 by the young Bellori (as a preface to Baglione's Vite de'Pittori) in which he classified modern artists according to the scheme of the three Graces. In the poem, Bellori devoted a complete strophe to each of the artists he would choose as "governors" thirty years later, describing his particular genius and relating him to one of the Graces.

The same kind of classification was used by the poet Antonio Bruni, who in Le Tre Gratie (1630) divided poetry into three broad categories: lyric, heroic, and moral and sacred. Each of these categories is governed by one of the Graces. Bruni asserts that his aim is to classify poetry with greater clarity and avoid the confusion generated by the mixture of different genres in a single poem.

A study of the emblematic etchings that precede the biographies confirms this analysis. Apparently the Idea, which was to become so famous in European academies of art, was added to the work very late, almost at the moment of printing. This late addition was the cause of some confusion at the printer's desk, with the result that this etching was misplaced in the text. Nevertheless the scheme of the programme appears very clearly. The three governors are associated with three concepts: Theoria, Praxis, and Conceptus-Imaginatio. The other three etchings in each group are related to the main theme and explain it. This figurative complement to the biographies testifies to the importance and vitality of such figurative and theoretical sources as Testa's Liceo della Pittura, also designed in the late 1630s. The iconographic analysis of the etchings shows that Bellori apparently adhered closely to the three-level educational programme defined by Testa for the perfect painter, seeking at the same time to reconcile schools and styles of the modern period in his Ideal Temple of Painting. Despite what has often been said, it was not his intention to oppose Poussin to Caravaggio, nor did he aim to write mere biographies of individuals.
Tous droits réservés (C) UAAC-AAUC (University Art Association of Canada | Association d'art des universités du Canada), 1987
Ce document est protégé par la loi sur le droit d'auteur. L’utilisation des services d'Érudit (y compris la reproduction) est assujettie à sa politique d'utilisation que vous pouvez consulter en ligne.

https://apropos.erudit.org/fr/usagers/politique-dutilisation/ 


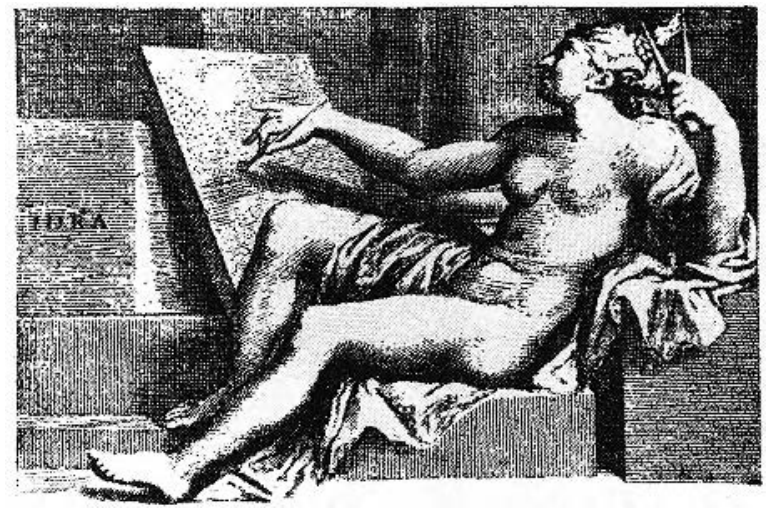

FicilRe 39. Fntourage de Ch. Errard, Idea, vignette emblématique pour G. P. Bellori, Le Vite de'Pittori, Scultori e Architett moderni (Rome, 1672), burin, $70 \times$ $105 \mathrm{~mm}$. (Photo d'après G. P. Bellori, Vite, 'I Iurin, 1976).

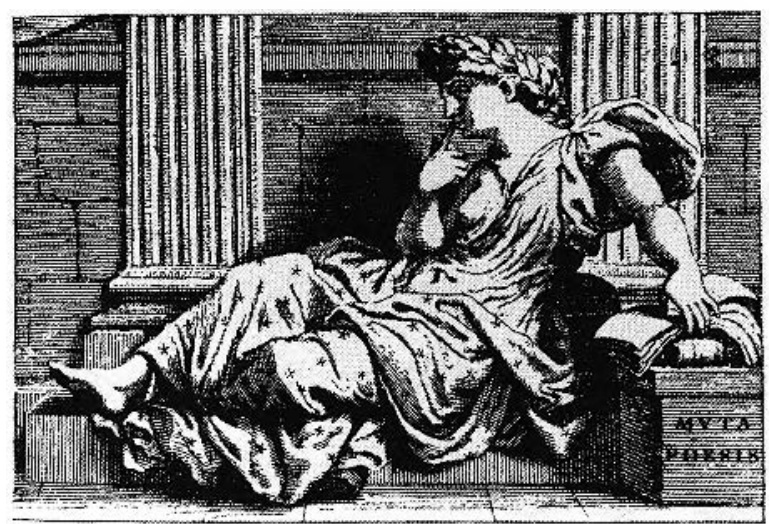

Fiolere 11. Entourage de Ch. Frrard, Muta poesis, vignctte cmblématiquc pour (;. P. Bcllori, Le Vite de'Pittori, Scultori e Architetti moderni (Rome, 1672), burin, $70 \times$ $105 \mathrm{~mm}$. (Photo daprès G. P. Bellori, Vite, Turin, 1976).

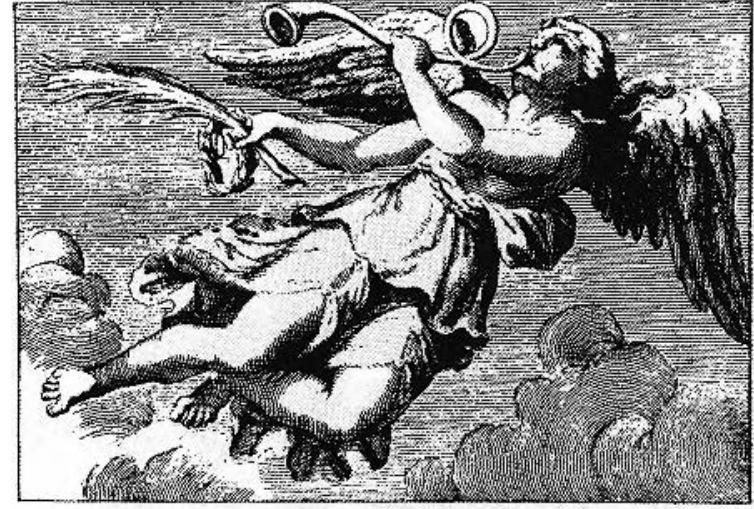

Figuri: 40. Entourage de Ch. trrard, La fama buona, vignette emblématique pour G. I'. Bellori, Le Vite de'Pittori, Scultori e Architetti moderni (Rome, 1672), burin, $70 \times$ 105 mm. (Photo d'après ('. P. Bellori, Vite, Turin, 1976).

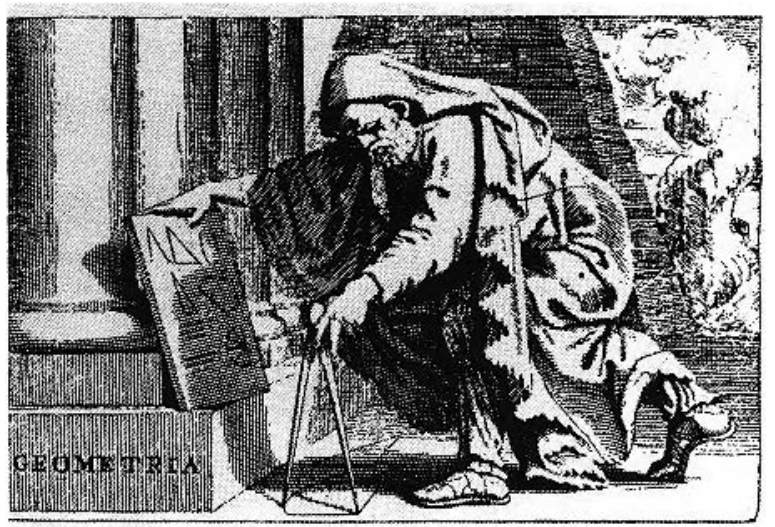

Figlek 42. Entourage de Ch. Errard, Geometria, rignette cmblomatique pour G. P. Bellori, Le Vite de'Pittori, Scultori e Architetti moderni (Rome, 1672), burin, 70 $\times$ $105 \mathrm{~mm}$. (Photo d’après (y. P. Bcllori, Vite, Turin, 1976). 


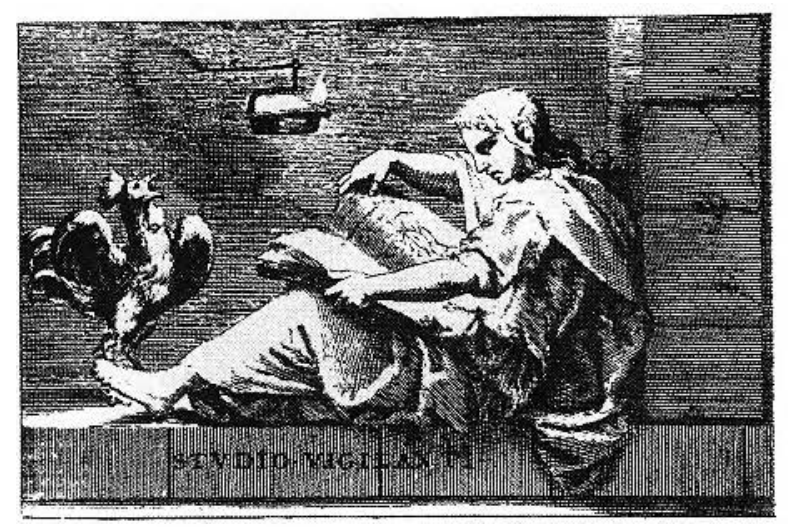

Figurf 43. Entourage de Ch. Errard, Studio vigilanti, vignette emblématique pour (;. P. Bellori, Le Vite de'Pittori, Scultorie Architetti moderni (Romc, 1672), burin, $70 \times$ $105 \mathrm{~mm}$. (Photo d'après (G. P. Bellori, Vite, Turin, 1976).

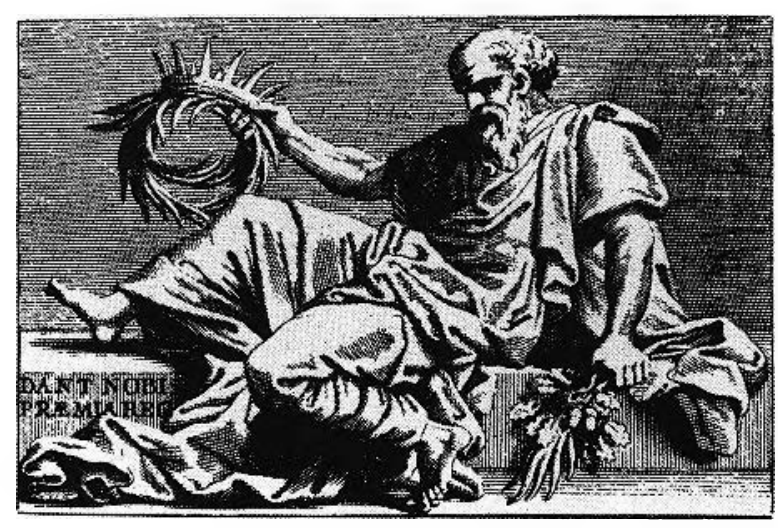

Figure 45. Entourage de (h. Errard, Dant nobis praemia reges, vignctte emblématiquc pour G. P. Bellori, Le Vite de'Pittori, Scultori e Archiletti moderni (Rome, 1672), burin, $70 \times 105 \mathrm{~mm}$. (Photo d'après (;. P. Bellori, Vite, Turin, 1976).

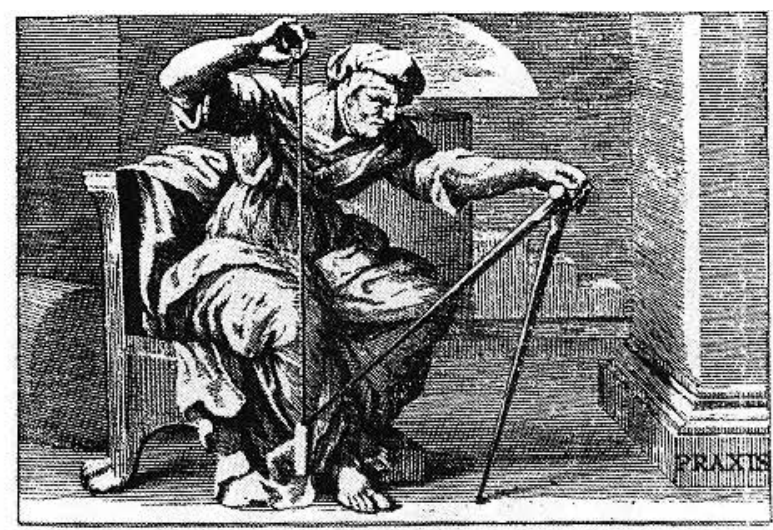

ligure 44. lintourage de Ch. Errard, Praxis, vignelte cmblématique pour (3. P. Bcllori, le Vite de'Pittori, Scultori e Architetti moderni (Rome, 1672), burin, 7()$\times$ $105 \mathrm{~mm}$. (Photo d'après G. P. Bellori, Vite, Turin, 1976).

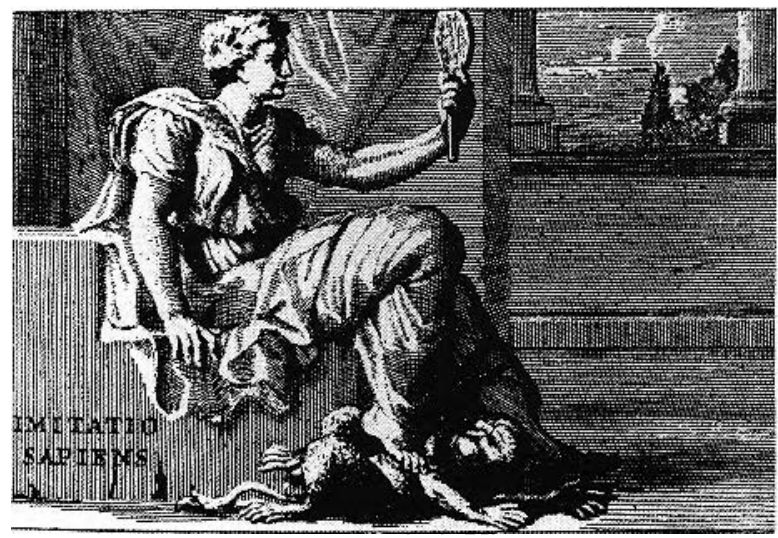

Figure 46. Fntourage de Ch. Errard, Imitatio sapiens, vignette emblématique pour G. P. Bellori, Le Vite de'Pittori, Scultori e Architetti moderni (Rome, 1672), burin, 7()$\times$ $105 \mathrm{~mm}$. (Photo d'après G. P. Bellori, Vite, Turin, 1976). 


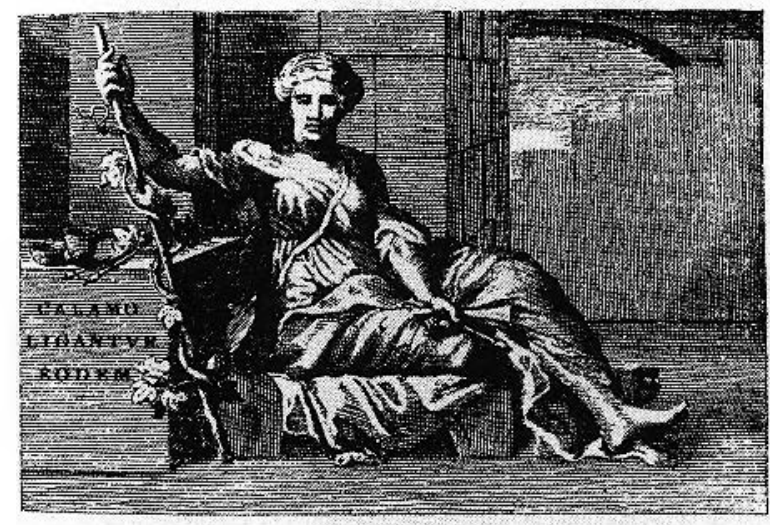

Ficiure 47. Entourage de Ch. Errard, Calamo ligantur eodem, vignette emblématique pour (;. P. Bellori, Le Vile. de'Pittori, Scultori Architetti moderni (Rome, 1672), burin, $70 \times 105 \mathrm{~mm}$. (Photo d'après G. P. Bellori, Vite, Turin, 1976).

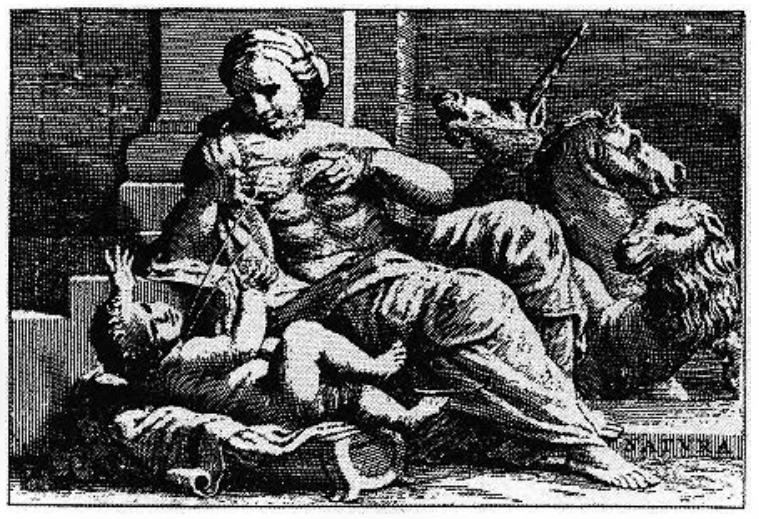

Ficotr: 49. Entourage de Ch. Errard, Natura, vignetue emblématique pour (. P. Bellori, Le Vite de'Pittori, Scultori e Architetti moderni (Rome, 1672), burin, 70 $\times$ $105 \mathrm{~mm}$. (Photo d'après G. P. Bellori, Vite, Turin, 1976).

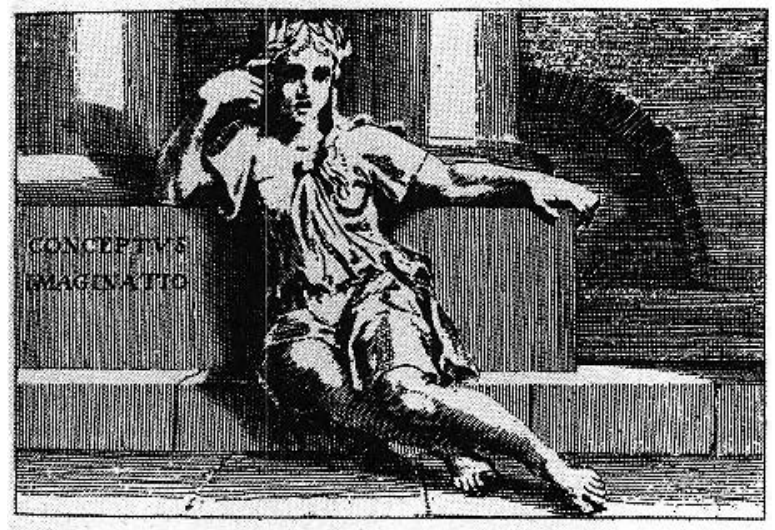

Figure 48. Fntourage de (ih. Frrard, Conceptus imaginatio, vignctte emblématique pour G. P. Bcllori, Le Vite de'Pittori, Scultori e Architetti moderni (Romc, 1672), burin, $70 \times 105 \mathrm{~mm}$. (Photo d'après G. P. Bellori, Vite, Turin, 1976).

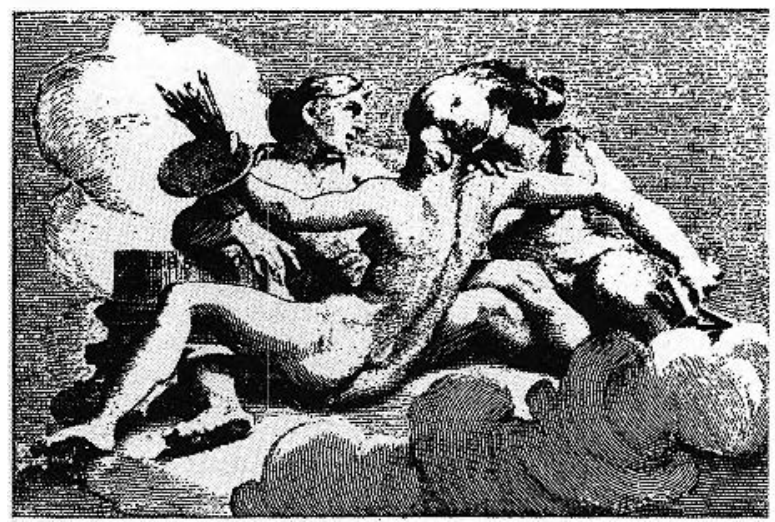

Figle jo). lintourage de (il. Frrard, Tres sorores, vignette emblématique pour (;. P. Bellori, Le Vite de'Pittori, Scultori e Architetti moderni (Rome, 1672), burin, $70 \times$ $105 \mathrm{~mm}$. (Photo d'après (. P. Bcllori, Vite, Iurin, 1976). 


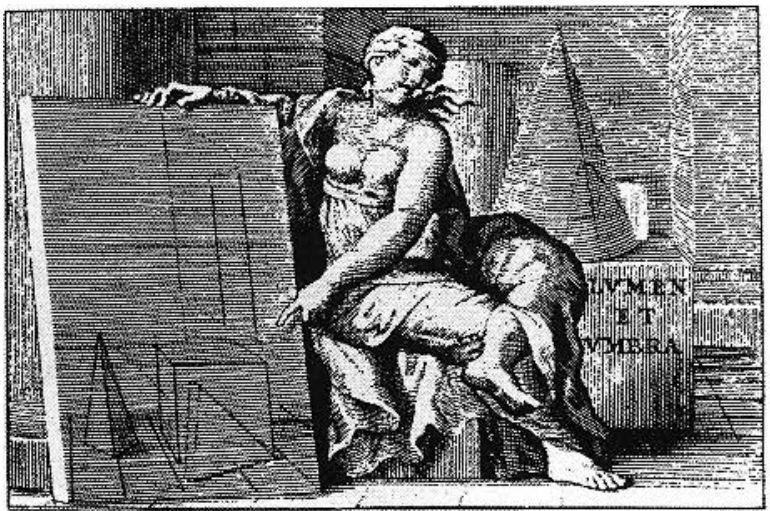

lisure jl. Entourage de Ch. Errard, Lumen et umbra, vignette emblématique pour G. P. Bellori, Le Vite de'l'ittori, Scultorie Architelti moderni (Rome, 1672), burin, 7()$\times$ 105 $\mathrm{mm}$. (Photo d'après G. P. Bellori, Vite, Turin, 1976).

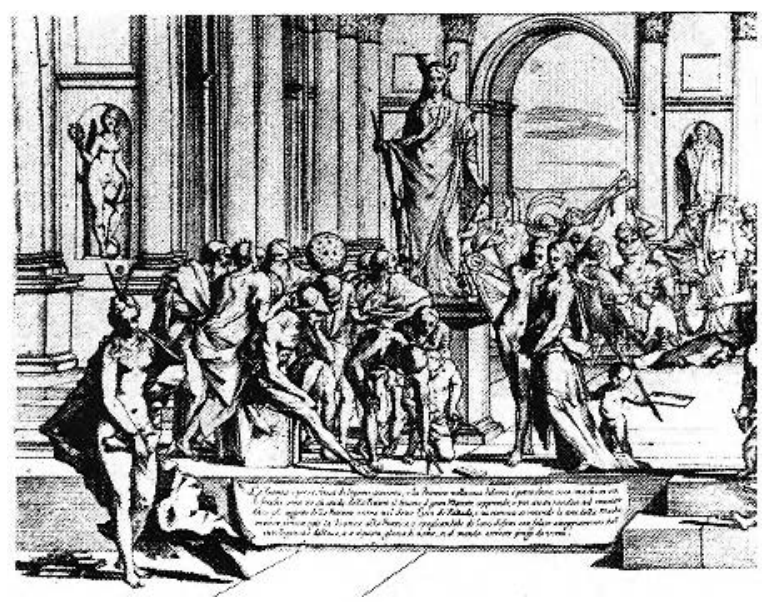

Figure 53. Détail de la Fig. 52: Theoria.

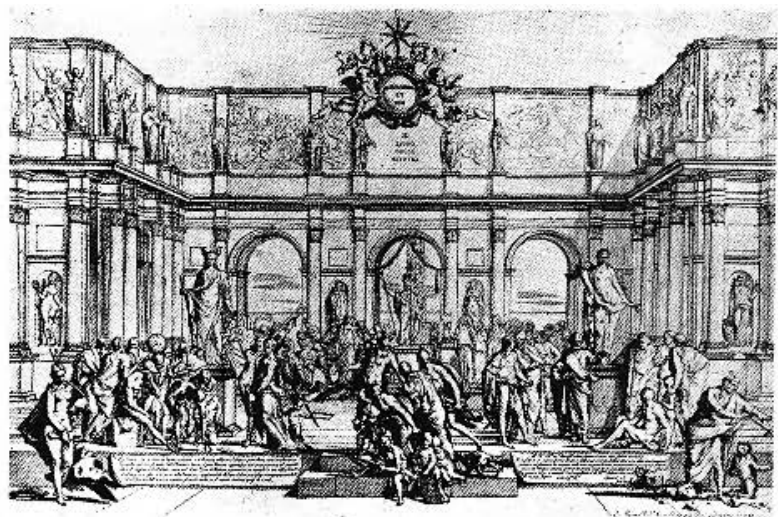

Figure 52. P. 'Testa, I/ Liceo della Pittura, fin des années 1630. burin. $473 \times 729 \mathrm{~mm}$. (Photo d'après The Illustrated Bartsch, xiv, 158 et 159)

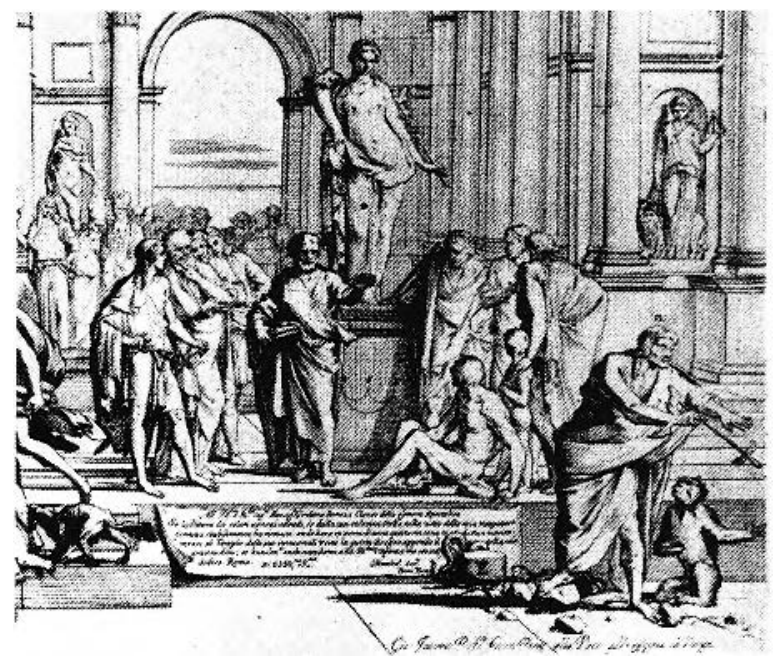

Figure 54. Détail de la Fig. 52: Prattica. 

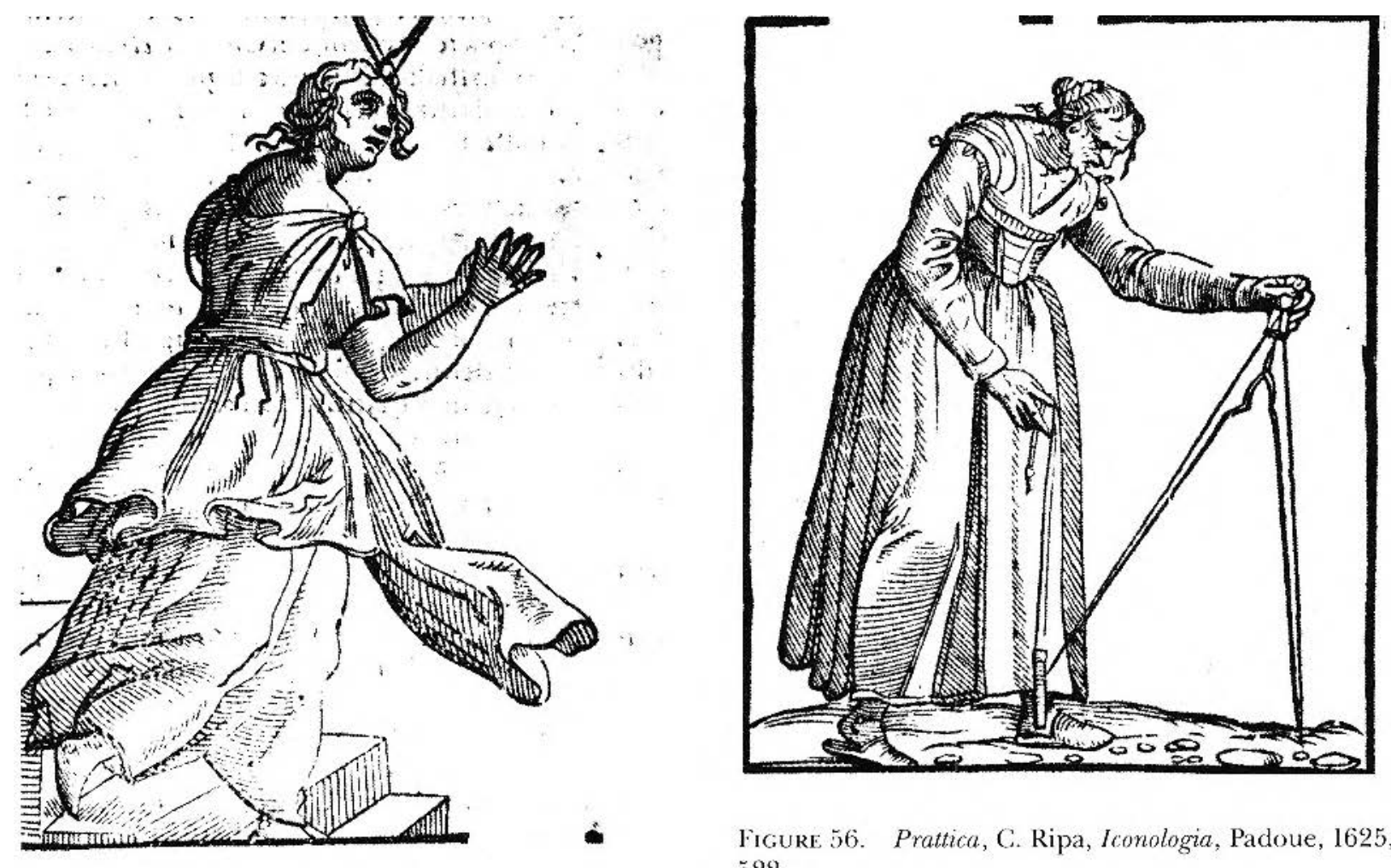

Figure 56. Prattica, C. Ripa, Iconologia, Padoue, 1625, 522 .

Figure 55. Theoria, C. Ripa, Iconologia, Padoue, 1625, 666.

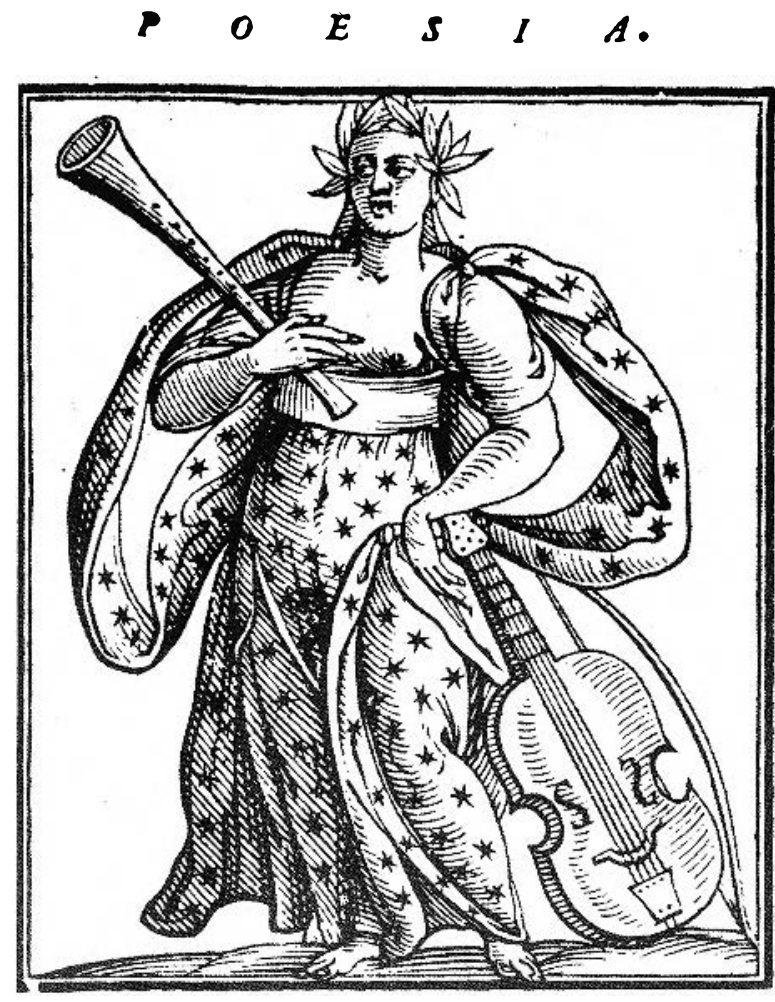

Figlre 57. Poesia, (. Ripa, loonologia, Padoue, 1611, 431.

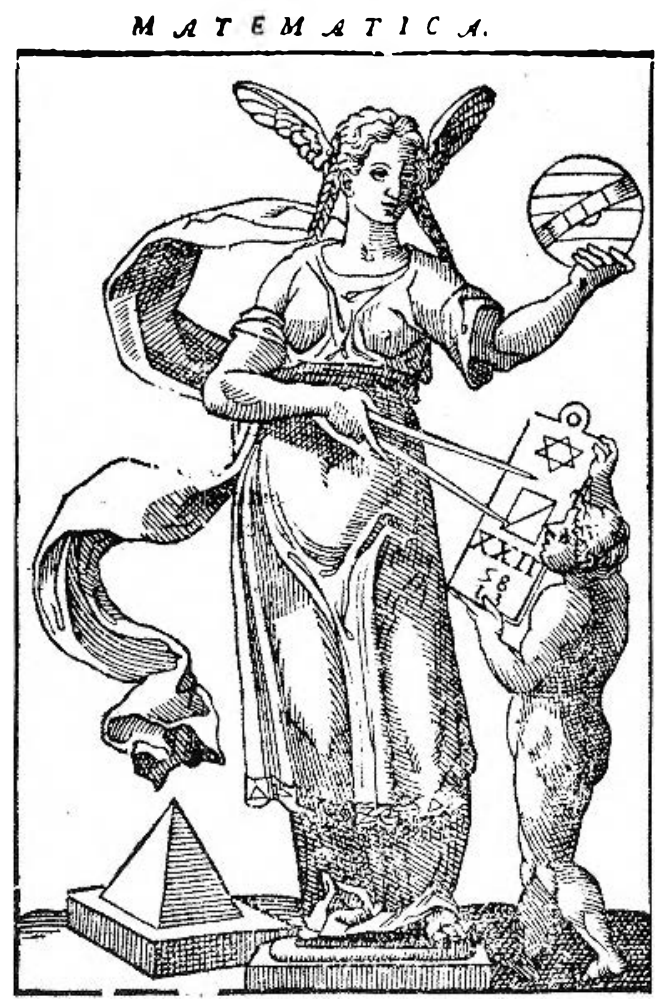

FIcitrt: 58. Matematica, C. Ripa, Iconologia, Rome, 1603, 308. 
$\begin{array}{llllllllll}I & N & \mathcal{T} & E & L & L & E & \mathcal{T} & \mathcal{T} & 0 .\end{array}$

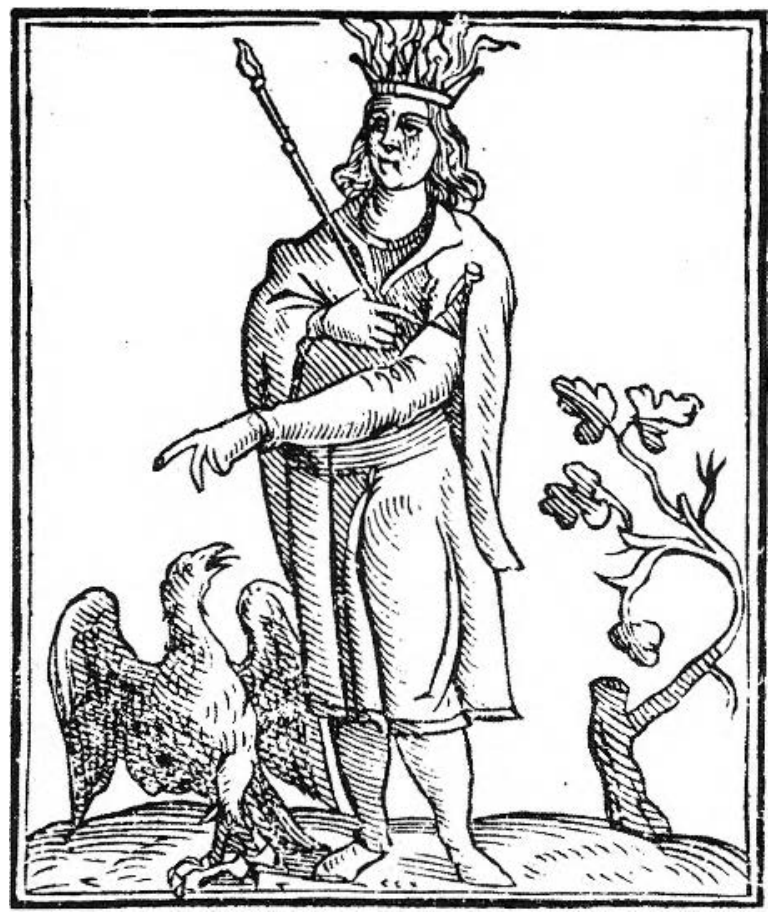

Ficicre 59. Intellello, C. Ripa, Icomologia, Padoue, 1611. 258.

QVINTA

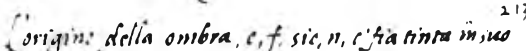

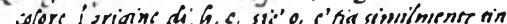

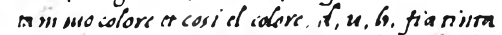
neicuiore fol pe piveche nasieda lui elombra dei miangolo, z, ky, fia nirm neliolore di.a. berclie deriua da cris. 1 .

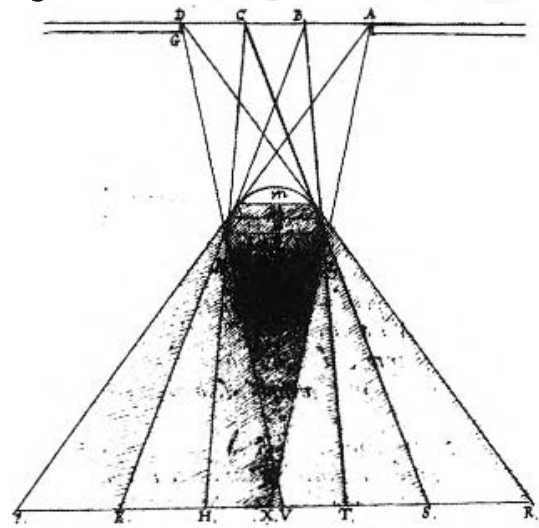

f.g, if prime grado di lame berche fuiti alumi

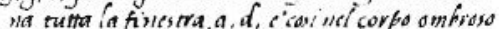

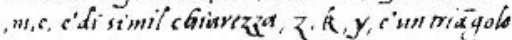

Ficiurr 66. Léonard de Vinci (d'après), dessin d'optique, Codex L'rbincas Latinus, 127(), fol. 21.3r. (Photo d'après Leonard de Vinci, Treatise on Painting, ed. A. Philip .Mc.Mahon, Princeton, 1956, I1, fac-similé).
$F V R O R \quad P O E T I C O$.

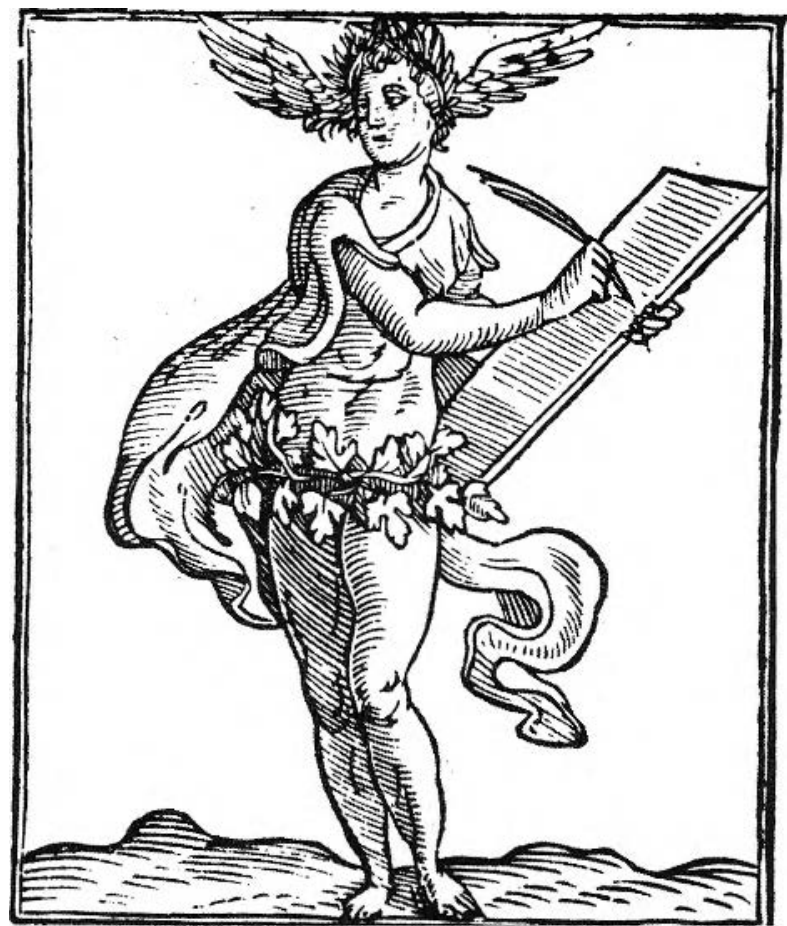

I'icier 60. Furor Portico, C. Ripa, Iconologia, Padouc, 16111. 191.

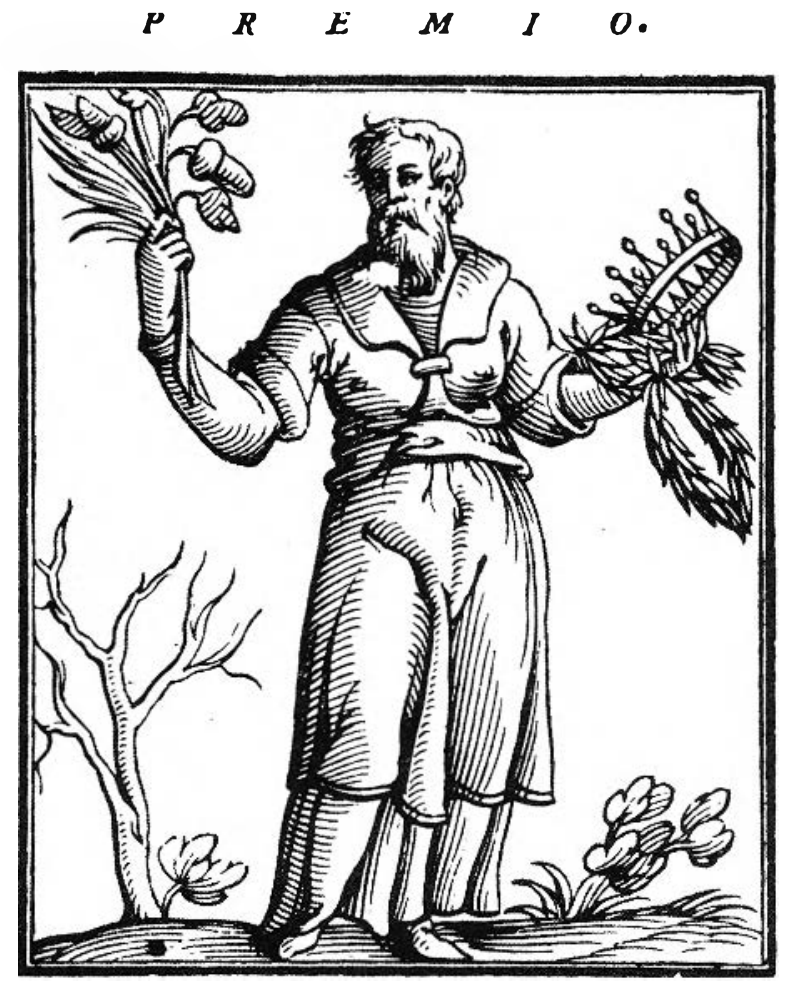

Fisile 61. Premio, (: Ripa, Iconologia, Padoue, I611, 437. 


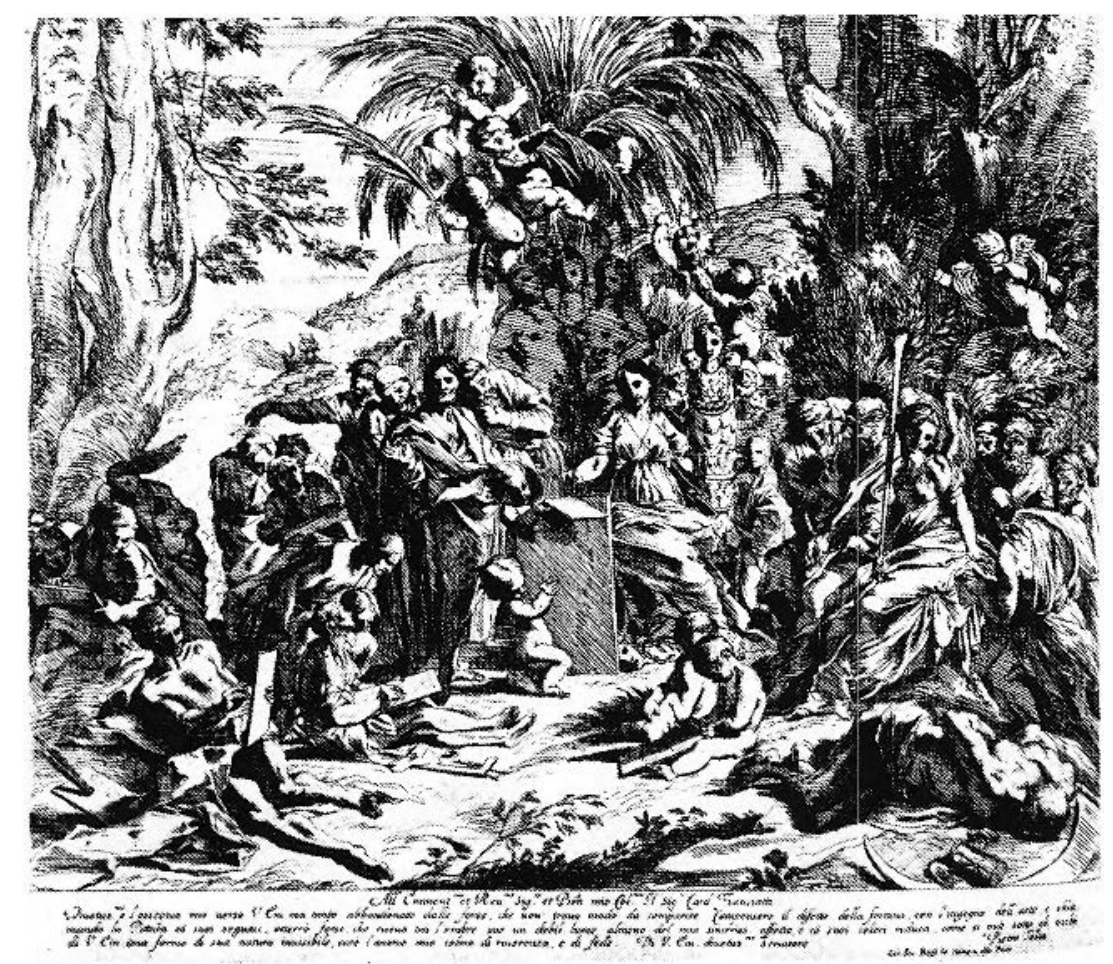

Fic;l'RE 62. P. lesta, Allégorie de la peinture, fin des années 1630, La peinture assise au milieu des plus habiles imaîtres de l'art, $262 \times 325 \mathrm{~mm}$. (Photo d'après The Illustrated Bartsch, xLv, 15l).

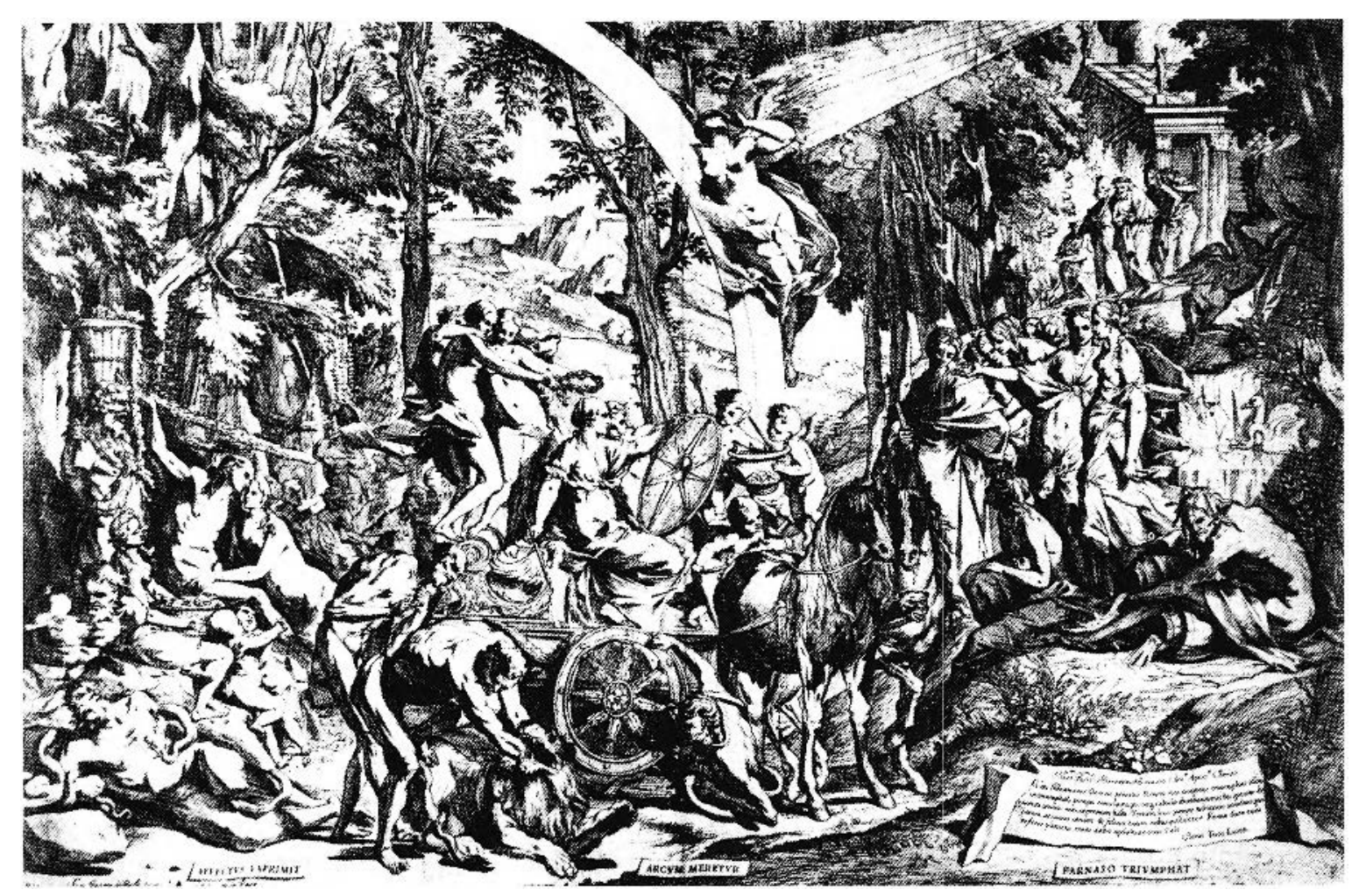

Figl're 63. P. Testa, Le triomphe de la peinture sur le P'arnasse, fin des annécs $1630,475 \times 723 \mathrm{~mm}$. (Photo) d'après The Illustrated Bartsch, xi.v, 16()-fil). 


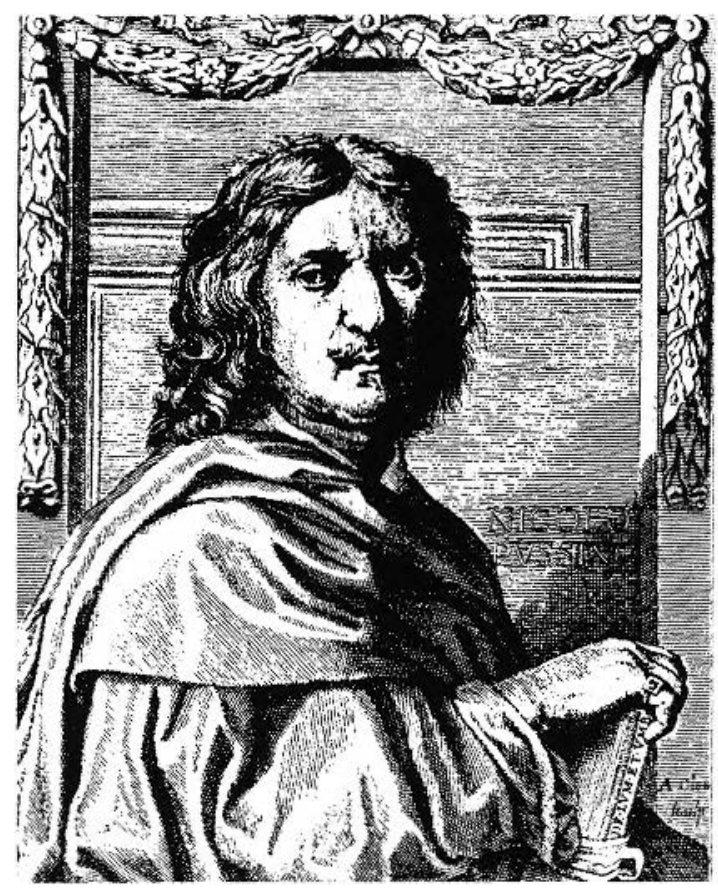

Ficiurl: 64. Fintourage de Ch. Errard, Nicolas Poussin (d'après l'autoportrail de 1650, Paris, Louvr(), burin pour l'édition de G. P. Bellori, Le Vite de'Piltori, Scultori e Architetti moderni (Rome, 1672), $131 \times 105 \mathrm{~mm}$. (Photo d'après G. P. Bellori, Vite, Turin, 1976).

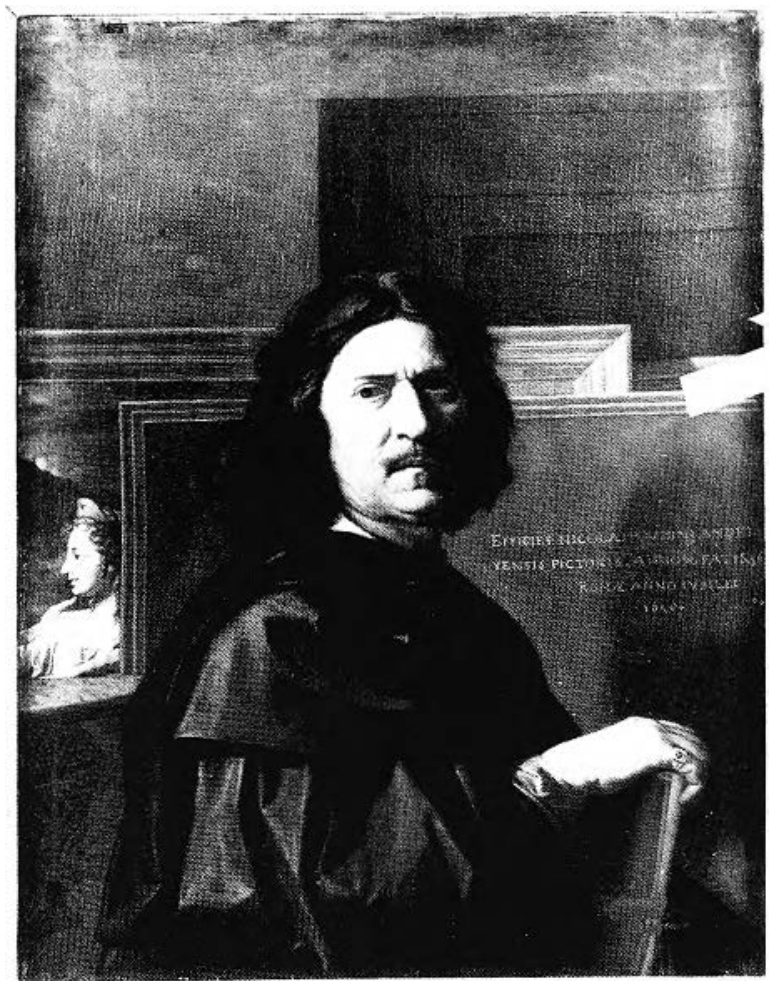

Ficilk1: 65. N'. P'oussin, Autoportrait, 1650, huile sur toile, P’aris, Lourre. Cliché des Musées Nationaux. 


\title{
Les Vite de 1672 de Bellori: hypothèses de reconstitution du programme iconographique et théorique*
}

\author{
JEAN-FRANÇOIS LHOTE
}

Université de Montréal

This article presents the evidence for a tentative reconstruction of the theoretical and iconographic programme of Bellori's Vite. The author seeks to show that Bellori's biographies are arranged according to a programmatic schemc probably conceived in the late 1630) or early 1640s, during the biographer's youth, and that this programme is inspired by Lomazzo's Temple of Painting and the theory of Governors.

Published in 1672, the Vite present the lives of twelve artists, following - up to a point - the chronological order of their deaths, as was the rule in that literary genre. However, three of them (Annibale Carracci, Caravaggio, and Domenichino) are out of chronological order, and at the same time divide the whole group into three equal parts. This anomaly is explained by a pocm published in 1642 by the young Bellori (as a preface to Baglione's Vite de'l'ittori) in which he classified modern artists according to the scheme of the three Graces. In the poem, Bellori devoted a complete strophe to each of the artists he would choose as "governors" thirty years later, describing his particular genius and rclating him to one of the Graces.

The same kind of classification was used by the poct Antonio Bruni, who in Le Tre Gratie (1630) divided poetry into three broad categories: lyric, heroic, and moral and sacred. Fach of these categories is governcd by one of the Graces. Bruni asserts that his aim is to classify poctry with greater clarity and avoid the confusion generated by the mixture of different genres in a single pocm.

A study of the emblematic etchings that precede the biographics confirms this analysis. Apparently the Idea, which was to become so famous in European academies of art, was added to the work very late, almost at the moment of printing. This late addition was the cause of some confusion at the printer's desk, with the result that this etching was misplaced in the text. Nevertheless the scheme of the programme appears very clcarly. The three governors are associated with three concepts: Theoria, Praxis, and Conceptus-Imaginatio. The other three etchings in each group are related 10) the main theme and explain it. This figurative complement to the biographics testifies to the importance and vitality of such figurative and theorelical sources as Testa's Liceo della Pittura, also designed in the latc 1630s.

The iconographic analysis of the etchings shows that Bellori apparently adhered closely to the three-level educational programme defined by Testa for the perfect painter, seeking at the samc time to reconcile schools and styles of the modern period in his Ideal Temple of Painting. Despite what has often been said, it was not his intention to oppose Poussin to Caravaggio, nor did he aim to write mere biographies of individuals.
La question étudiée ici a déjà été posée par les éditeurs, biographes et commentateurs de Bellori : est-ce que les Vite de'Pittori, Scultori, e Architetti

\footnotetext{
* Cet article est unc partie (remaniée) d'une étude plus vastc. Afin de respecter des limites raisonnables, je ne présente ici que l'essentiel de la question. Volontairement, j’ai laissé cle côté certains problèmes importants: la seconde partie des Vite. les procédés cle transformation par Bellori clu modèle biographique de Vasari, l'évolution de la notion d'école chez Bcllori, etc.
}

moderni de Giovan Pietro Bellori, publiées à Rome en 1672, sont ordonnécs selon un programme iconographique et théorique cohérent et à peu près systématiquc? ${ }^{1}$ Autrement dit, y a-t-il dans cet.

I On pourra consulter notamment sur cette question : Kenneth Donahue, "The Ingenious Bellori", Marsyas, III (1946), 107-38. Du même duteur on verra l'article "Bellori Giovanni Pietro ", dans Dizionario biografico degli Italiani, vil (1965), 781-89. On verra aussi l'article de Anna Pallucchini, 
ouvrage une architecture symbolique, un ensemble ordonné de règles qui président au choix des biographies, à leur agencement, à leur composition même, bref, peut-on admettre l'idée que cet ensemble de douze biographies n'est pas la pure transcription du hasard historique mais bien l'image d'un Temple idéal de la Peinture, à la manic̀re de Lomaz:o, et le projet d'une académie de la Peinture idéale? Je mentionne ici Lomazzo parce que Bellori s'est largement inspiré du programme de l'Idea del Tempio della Pittura publié en 1590 et qu'il a employé comme préface pour l'ouvrage de 1672 une conférence qu'il avait fait lire en 1664 à l'Académic de Saint Luc, la célèbre Idea del Pittore, dello Scultore, e dell'Architetto.

Editeurs et biographes de Bellori ont soupçonné l'existence d'un programme sans parvenir à le définir précisément. Une telle découverte aurait été d'un immense intérêt pour l'intelligence de la théorie idéaliste au xvir ${ }^{\mathrm{e}}$ siècle, car Bellori est un personnage de premier plan. Selon Schlosser, il fut "l'historiographe de l'art le plus important non seulement de Rome mais d'Italie et même de toute l'Europe au xvir ${ }^{e}$ siècle ". Et il ajoute : "son influence et sa valeur dépassent de loin les limites de son propre champ " ${ }^{2}$. Pour Panofsky il fut à son époque le plus célèbre savant versé aux choses de l'art ${ }^{3}$. Avant tout il était antiquaire, remarquable par sa salence, et lin connaisseur. Depuis là fin des années 1660 Bellori était commissaire aux antiquités de Rome, un poste considérable, avant de devenir antiquaire et bibliothécaire de Christine de Suède ${ }^{4}$.

L'ouvrage même qu'il publie en 1672 deviendra le canon et la règle des académics artistiques en Europe jusqu'à la fïn du xix $x^{\mathrm{e}}$ siècle ou presque, et agira au passage de façon déterminante sur l'orientation d'un esprit aussi distingué que celui de Winckelmann. Il s'agit d'un gros livre de plus de 460 pages. Il est composé d’une dédicace à Colbert, d'une adresse au lecteur suivie d'un exergue puisé aux Eicones de Philostrate et surtout, en guise de préface, de la conférence de l'Idea. Suivent douze biographies d'artistes qui représentent le gros de l'ouvrage (440 pages environ sur 462). Il

"Per una situazione storica di Giovan Pietro Bellori", Storia dell'arte, xu (1971), 285-95. Sur la composition de l'ouvrage par Bellori: Fugenio Battisti, "Il Bellori come critico ", Le vite de'pultori (Gènes, 1967), vii-xxx. Sur l'apparente inexistence d'un programme cohérent on verra: Giovanni Previtali, "Introduzione ", dans Le vile de'pittori ('Turin, 1976), ix-lx. C'est à cette édition de l'ouvrage de Bcllori que nous ferons référence dans la suite du texte, en utilisant la mention abrégée: Vite.

2 Julius Schlosser Magnino, La letleratura artistica, 2“ éd. (Vicnne, 1956), 463-64.

3 Erwin Panofsky, Idea: A Concept in Art Theory (New York, 1968), 105.

4 Donahue, "The Ingenious Bellori ", 786. s'agit des frères Annibale et Augustin Carrache, de l'architecte D. Fontana, du Baroche, du Caravage, de Rubens, de Van Dyck, de Duquesnoy, du Dominiquin, de Lanfranc, de l'Algarde et de Poussin: un architecte, deux sculpteurs et neuf peintres. Chaque biographie est précédée d'un portrait gravé de l'artiste et, tout comme la préface, d'une vignette gravée à caractère emblématique associée à un motto (Figs 39 à 51). Qui dit emblème laisse entendre programme et c'est sur ces vignettes que s'est portée l'enquête des biographes et des éditeurs, mais sans succès.

Nous savons que Bellori choisit volontairement et après de longues réflexions les douzes artistes qui devaient figurer dans l'ouvrage. Dans une lettre du 16 mars 1669 adressée à l'antiquaire florentin Carlo Roberto Dati, il écrit :

Les vies des peintres que j'écris en ce moment ne sont ni celles des Romains ni d'aucunc autre région en particulier. J'ai choisi quelques artistes - très peu - selon mon pauvre jugement. Je doute pourtant, dans ce petit nombre, de m'être avancé car notre époque est chiche et ni en peinture, ni en poésie, ne doit-on estimer la médiocrité

Si l'idée de choisir quelques artistes peut nous sembler aller de soi aujourd'hui, il n'en allait pas de même au xvi ${ }^{\mathrm{e}}$ siècle. Le romain G. B. Passeri, lui-même auteur de biographies d'artistes et qui écrit en même temps que Bellori, a jugé bon, dans son adresse au lecteur, de désapprouver le parti pris de son concurrent.

Les astrologucs ne parlent pas que de Jupiter, Saturne ou Mercure [...]. Ils ont distribué les étoiles en six grandeurs et font autant de cas de celles de la première que de celles de la dernière [...]. Tous les artistes qui ont laissé quelque renom et quelque témoignage de leur valeur ont le droit d'apparaître dans un ouvrage historique ${ }^{6}$.

5 " [...] le vite de'Pittori, che io vado scrivendo non sono quelle ne di Romani ne d'altra particolare regione, ma ne ho'scelti alcuni pocchi secondo il mio debole giudicio. Dubito nondimeno nel poco numero di essermi avanzato per la scarsità di nostri tempi, ct la mediocrita cosi nella pittura, come nella poesia non deve essere in istima " (Lettere di diversi a Carlo Roberto Dati, Florence, Bibl. Na». Cent., Racc. Baldovinetti, Misc. 258, fasc. II, fol. 33).

6 . Gl'Astrologi nelle loro fïgure non fanno mentione solamente di Saturno, di Giove, di Marte, del Sole, di Venere, di Mercurio c della Luna, ancor lc maggiori, le più luminose, e le stelle più fisse; ma havendole tutte distribuite in sei grander.zc, tanto fanno caso di quelle della prima, quanto di quelle dell'ultima sorte; [...] Con questa imitatione, per formare una compita Istoria, io ho voluto trattare tanto nella Pittura, quanto nella Scoltura, et Architettura di tutti quelli, li quali à tempo loro hanno riportato qualche grido del loro valore, e lasciato qualche onorevole memoria del nome loro; ma ho scritto di ciascheduno à proportione della loro propria qualità, nè ho voluto defraudarc nessuno di quella parte, che se gli deve" (Jacob Hess, ed., Die Künstlerbiographien von Giovanni Battista Passeri, 2 vols [Leipzig et Vienne, 1934], 5). 
En effet, un historien qui écrit la biographie des artistes modernes est d'abord un chroniqueur. C'est ainsi que l'entend Passeri. Vasari, Borghini, Baglione ont écrit, pour ainsi dire, les Fastes de l'art moderne. Il faut reprendre le fil exactement où le prédécesseur l'a abandonné, sans laisser de vide. L'un des projets de page titre prévu par Passeri pour son ouvrage mentionne qu'il commencera là où Baglione a laissé (Baglione qui luimême avait repris la chronique de Vasari). C'est pourquoi Passeri commencera en 1641 pour venir jusqu'en $1673^{7}$.

Pour classer ces artistes on procèdera de la façon la plus simple, en suivant l'ordre chronologique qui est celui de la chronique. "L'ordre que j'ai choisi, dit Passeri, est celui du temps, je me suis réglé sur les années suivant exactement leur cours ${ }^{8}$. En fait, la règle de distribution des artistes dans la chronique est le classement par l'année de la mort. Second classement superposé au premier: on distribue les artistes par chapitres selon le pontificat où ils ont travaillé et où ils sont morts.

La règle suivante est tout aussi absolue: on ne parle que des morts. Seule la mort donne le droit d'être recensé dans la chronique: De vivis non disputandum. C'est cette règle qui permet à Bellori en 1672 de ne pas parler du Bernin, à son grand soulagement.

Dernière question: comment décrire les oeuvres des artistes? Réponse : par ordre chronologique et simplement. C'est du moins la réponse de Passeri.

Les oeurres décrites dans mon livre sont classées par ordre chronologique; je les décris exactement, sans élégance, sans recherche ni éloquence car je ne suis pas un ćrudit... Mon oeuvre n'a pas besoin de rhćtorique car je traite une Histoire d'actions simples qui exige pureté

7 Le texte du projet de page titre est le suivant:

"Il tempo distruggitore

Delle Vite de

Pittori Scultori et

Architetti

Seguitando dove lasciò il Cavaliere Giovanni Baglioni, che unissi al Vasari [...]" (Hess, ed., Die Künstlerbiographien, Planche 1 .

8 "L'ordine che io ho tenuto, è stato qucllo del tempo, perche mi sono regolato dagl'anni, seguendo osservatamente il corso loro " (Hess, ed., Die Künstlerbiographien, 5).

9 "Del rimanentc, se brami (accorto lettore) una florida locutione, una eleganza di frase, et una nobiltà d'eloquenza, qualità convenienti ad uno erudito scrittore, ti converrà sof frire in patienza, se in me trovi questo mancamento. Due ragioni ti addurrò per mia discolpa, c difesa : una che la occasione non solo non richiede, ina proibisce rigorosamente il maneggio d'una dicitura amena, florida, e traslata, perche io tratto una Istoria d'attioni semplici, che vuol purità nella spiegatura; e non un 'Trattato d'opere eroiche, c gloriose (li gran Personaggi, al quale si conviene maestà, et grandezza "(Hess, ed., Die Künstlerbiographien, 5). de l'exposé. Je n'écris pas un Traité d'oeuvres hérö̈ques et glorieuses de grands personnages à qui convient majesté et grandeur?

Le grand mot est lâché : l'ouvrage de Bellori n'est pas une Histoire mais un Traité où l'éloquence joue un rôle majeur.

A Paris, le rapporteur du Journal des Sçavans (en date du lundi 7 décembre 1676) voit les choses d'un autre oeil et estime que Bellori a évité les deux défauts majeurs où tombent les "auteurs de recueils de vies de peintres ", à savoir le campanilisme et la confusion ${ }^{10}$. Premier défaut le campanilisme de Vasari et Ridolfi. Bellori n'est pas le défenseur d'une école particulière de peinture, il s'en défend dans la lettre citée plus haut. Les artistes dont il traite viennent de toutes les régions, de tous les climats et des différentes écoles: Parme (Lanfranc), les Flandres (Rubens, Van Dyck, Duquesnoy), Bologne (Augustin et Annibale Carrache, le Dominiquin, l'Algarde), Urbin (le Baroche), la Lombardie (Caravage, D. Fontana), la France enfin (Poussin). Second défaut : la confusion de Baglione.

[...] la confusion des moindres apprentis avec les plus excellens maistres: [...] le Baglione a ramassé indifféremment plus de deux cens peintres, sculpteurs et architectes tant bons que mauvais dont les noms ont aussi pcu duré que les ouvrages ${ }^{11}$.

Ce choix des "plus excellens maistres " devait mener presque inévitablement à l'héroïsation du sujet dont parle Passeri. En effet, non seulement l'héroïsation est à la mode dans le milieu français que fréquente Bellori, mais sa formation d'archéologue et ses goûts littéraires le poussent à vouloir décrire la perfection. Dans l'adresse au lecteur des Vite il avoue qu'il a failli ne rien écrire faute de rencontrer l'artiste parfait. Mais après tout, ajoute-t-il, les meilleurs artistes grecs eux-mêmes furent très éloignés de la perfection. C'est pourquoi il s'est résolu à choisir quelques modernes ${ }^{12}$. Coquetterie de rhéteur? Oui, mais aussi conviction profondément établie chez lui que la biographie est de l'ordre de l'éthique et que le biographe doit donner au lecteur le désir d'imiter le modèle qu'on lui propose. Ce modèle doit faire partie des meilleurs. Cette conviction ne lui vient pas sculement de la fréquentation de la Poétique d'Aristote, mais de son guide en matière d'écriture historique, le traité si célèbre en son temps d'Agostino Mascardi, Dell'Arte historica ${ }^{13}$. Traitant des divisions de l'Histoire, Mascardi avait examiné le genre biogra-

\footnotetext{
10 Journal des Sçavans. Du Lundy 7 Decembre M.DC. LXXVI, 229-33.

11 Journal des Sçavans, 229

12 Bellori, Vite, 7

13 Agostino Mascardi, Dell'arte historica (Rome, 1636).
} 
phique et décidé que le modèle en était Plutarque, l'objectif de la biographie étant d'exprimer l'image du virtuoso, d'en dresser le portrait moral, d'en faire comprendre le caractère et la nature ${ }^{14}$.

On pourrait croire, toutefois, que Bellori ait voulu concilier chronique et histoire des héros. Les artistes apparaissent dans les Vite en suivant, semble-t-il, l'ordre chronologique. En fait, on constate à l'examen des distortions notables. A vrai dire, elles passeraient inaperçues sans l'aide de Passeri qui insiste tant sur l'ordre chronologique de son propre livre. Voici l'ordre d'apparition des artistes dans les Vite:

\begin{tabular}{lcc} 
Annibale Carrache & décédé en 1609 \\
Augustin Carrache & $"$ & 1602 \\
D. Fontana & $"$ & 1607 \\
Le Baroche & $"$ & 1612 \\
Caravage & \multicolumn{2}{c}{1609 (selon } \\
& $"$ Bellori, en fait 1610) \\
Rubens & $"$ & 1640 \\
Van Dyck & $"$ & 1641 \\
Duquesnoy & $"$ & 1643 \\
Le Dominiquin & $"$ & 1641 \\
Lanfranc & $"$ & 1654 \\
L'Algarde & $"$ & 1665
\end{tabular}

Le strict ordre chronologique serait respecté si trois artistes n'étaient déplacés dans la série, Annibale Carrache, Caravage et le Dominiquin. Ce déplacement est en même temps "régulier " puisqu'ils sont tous trois répartis de façon à diviser les douze artistes en trois groupes. Ce serait là détail sans grande importance si d'autres éléments ne venaient confirmer que cette erreur est volontaire et que nous sommes au coeur même du projet théorique de Bellori.

Curieusement, on peut constater que les trois artistes "déplacés" des Vite de 1672 avaient, trente ans auparavant, reçu de Bellori un traitement particulier dans le poème qu'il avait écrit et publié en guise de préface aux Vite de'Pittori de G. Baglione $(1642)^{15}$. Il est compréhensible que l'on n'ait pas attaché d'importance à ce poème. Bellori lui-même, dans son exemplaire de l'ouvrage de Baglione-exemplaire qui nous est parvenu - avait renié ce péché de jeunesse. En marge, il avait noté : "J'ai écrit ce poème lorsque j'étais jeune. Aujourd'hui il ne me plaît plus. Je

14 Mascardi, Dell'arte historica, $63 \mathrm{sq}$.

15 I.e Vile de'Pittori Scultori e Archiletti Dal Pontificato di Gregorio XIII del 1572. Insino à tempi di Papa Urbano Ottavo nel 1642, scritte da Gio. Baglione Romano, Roma, 1642. L'exemplaire annoté par Bellori (parmi d'autres) a été ré-édité en facsimilé par le R. Istituto d'archeologia e storia dell'arte à Rome en 1935. Cet exemplaire est conservé à la Bibliothèque de l'Accademia dei Lincci. l'avais fait imprimer parce que Tronsarelli m'en avait persuadé mais je n'y tenais pas ${ }^{16}$.

Ce poème intitulé Alla Pittura a douze strophes, la douzième n'étant qu'un tercet. Les deux premières strophes font l'éloge de la peinture; la troisième évoque la peinture antique; la quatrième la résurrection de la peinture à la Renaissance par le pinceau de Raphaël; la cinquième et la sixième évoquent les grands artistes du $\mathrm{xvr}^{\mathrm{e}}$ siècle, Léonard, Titien, Corrège, Zuccaro, le Baroche, le Cavalier d'Arpin, Borgiani. Les trois strophes suivantes $(7,8$ et 9$)$ sont consacrées chacune à un seul artiste: Annibale Carrache $\left(\mathrm{n}^{0} 7\right)$, Caravage $\left(\mathrm{n}^{0} 8\right)$ et le Dominiquin ( $n^{\circ} 9$ ). Les strophes 10 et 11 font l'éloge de Baglione et de Rome devenue le Parnasse. La strophe 12 est un simple final. Les termes du reniement de son oeuvre par Bellori invitent à supposer que bien du temps s'écoula avant qu'il ne relut son poème. En fait, il dut le faire au moment où préparant les Vite il relut Baglione et l'annota de remarques marginales parfois divertissantes et de jugements sur son prédécesseur. Ayant désavoué son poème, il reprit pourtant la plume et le corrigea: il biffa les strophes 10 et 11 d'éloge de Baglione. Le poème dès lors s'achevait sur l'éloge du Dominiquin. Et les seules strophes qu'il corrigea furent la 7 d'éloge d'Annibale et la 8 d'éloge du Caravage.

Ammira, de' Caracci alteri pregi

Quel di Natura emulator sublime

Annibal, che ne l'opre

Sembianze eterne, Idee Celesti exprime;

Che inentre arte discopre

Sovrana, e al mondo sola,

Tutti a le Gratie inuola

Gli honor, le glorie, le vaghezze, e i fregi;

E'l suo gran nome hor vola

Resa Cartago humile,

(F. Bologna immortal) dal Indo al Thile ${ }^{17}$,

\section{8}

Scorgi da Caravaggio il gran Michele

Dar ne l'opre a i colori, e vita, e albergo,

E con verace finto

Lasciare il ver de le sue larve a tergo

Non è, dipinto

Quel, che ne'lini sui

Mostra a le luci altrui.

Che non volgari effigio le tele;

Ma la Natura a lui

16 Dans la marge gauche de la première strophe: "Canzone da me fatta negli anni giovanili, che hora non mi piace niente: mandata in stampa più tosto a persuasione del Tronsarelli che per mia volontà ".

17 Dans le dernier vers Bellori a effectué la correction suivante: "Indo " a remplacé "Battro". 
Vive tempre concesse,

Suoi spirti diede, e in lor se stessa impresse ${ }^{18}$.

Guarda colui, ch'a le mort'ombre puote

Dar con mirabil'arte industre, e saggio,

E vita, e senso, e mente;

Nè già furtivo invola il Solar raggio

A l'alta rota ardente.

Con esso (o meraviglia)

Aglaia si consiglia.

Già fatta con le Suore a lui devote

Imitatrice, e figlia:

Zampieri il grande è questi;

E tu su'l Reno a noi, Felsina, il desti.

Si nous examinons le contenu de ces trois strophes nous pouvons constater deux choses. Premièrement, Annibale est caractérisé comme celui qui exprime dans ses oeuvres les Idées Célestes et les semblances éternelles. Caravage est caractérisé comme coloriste et comme représentant de la Nature. Le Dominiquin, enfin, donne aux ombres la vie, le sens et l'esprit. Deuxièmement, Bellori fait deux fois référence aux Grâces, une première fois à propos d'Annibale et une seconde lorsqu'il associe le Dominiquin à Aglaia, la Grâce qui représente la splendeur solaire. Il est aisé de suivre cette piste et de constater que ces trois strophes constituent une sorte de petit poème à la fin du grand et que le thème unificateur de ce petit poème est la réunion des trois Grâces, Euphrosyne, Thalia et Aglaia. Il s'agit des Grâces de la tradition ficinienne du Commentaire sur le Banquet de Platon ${ }^{19}$. Ficin avait défini la Beautédont l'âme se nourrit - comme la réunion des trois Grâces. Plus exactement, disait-il, la Beauté se déploie dans la trinité des Grâces, Splendeur, Viridité et Joie.

Il [Orphée] appelle Splendeur cette Grâce et cette beauté de l'âme qui est dans l'éclat de la vérité et de la vertu, Verdeur la douceur de la figure et de la couleur, car elle s'épanouit au plus haut dans la viridité de la jcunesse. Joie, enfin, le plaisir vrai, sain et durable que nous précure une mélodie musicalc ${ }^{20}$.

Pour Bellori, Annibale Carrache est gouverné par Euphrosyne, la Joie, et son art se situe dans la sphère des Idées et de l'Harmonie Céleste. Nous en aurons confirmation par l'étude des vignettes. Le Dominiquin est Aglaia, Splendeur. Caravage enfin est Thalia, couleur des figures, force vitale venue du monde souterrain du silence et de la

18 L.e vers original: "Suoi spirti diede e se medesima impresse " a été corrigé ainsi: "Suoi spirti diede e in lor se stessa impresse".

19 M. Ficin, Commentaire sur le Banquet de Platon, trad. françaisc par R. Marcel (Paris, 1978).

20) Ficin, Commentaire, 181-82. germination, Nature. Cette association Caravage-Thalia permet d'éclairer le sens que Bellori donne au concept de Nature et en même temps l'idée qu'il se fait véritablement du Caravage, et explique la réunion dans un même groupe de Caravage, Rubens, Van Dyck et Duquesnoy.

L'identification de Thalia à la force germinative était bien connue. Le musicologue vénitien F. Gafurius avait, dans son échelle harmonique, associé Thalia à l'univers inférieur et au silence, tout comme il avait associé Aglaia à l'univers apollinien de la splendeur solaire et Euphrosyne à la sphère céleste d'Uranie ${ }^{21}$. Pour Lomazzo, Thalia était liée à la lune, étant l'humeur par laquelle la terre ver$\mathrm{dit}^{22}$. Le philosophe néo-platonicien $\mathrm{F}$. Patrizi s'était expliqué un peu longuement sur cette humeur qui fait verdir. Dans un petit opuscule adressé en 1552 à Mariano Savello et intitulé Discours de la diversité des fureurs poétiques, il constatait que les muses ne sont que le nom des âmes, des forces qui meuvent les sphères célestes ${ }^{23}$. Chacune des âmes de ces planètes provoque en l'homme qui est soumis à son influx, une impression conforme à sa nature. La lune-Thalia génère en nous les affects et le désir d'engendrer que l'on assimile parfois à la fureur, comme elle gouverne la génération des animaux. Le soleil donne aux êtres la bonté et l'acuité des sens. Comme on voit nous ne sommes pas très éloignés de la magie astrologique qui fait le fond du Traité de Lomazzo.

C'est sans doute à Lomazzo que pense Passeri lorsqu'il dit de Bellori qu'il a écrit la vie des Maestri Primieri ${ }^{24}$. Tant dans le Trattato de 1584 que dans l'Idea del Tempio della Pittura, Lomazzo avait donné un modèle de critique artistique fort éloigné du modèle historique choisi par Vasari. Il avait, comme on sait, jeté les bases du relativisme critique. La constatation de la diversité des goûts et des talents l'avait amené à fonder son édifice critique non sur le nécessaire mouvement de l'Histoire mais sur une typologie des artistes. Aux trois

21 Franchinus Gafurius, Practica Musice (Venise, 1496). Sur Gafurius et son échelle harmonique on lira les commentaires - aujourd'hui classiques - d'Edgar Wind, Pagan Mysteries in the Renaissance (New York, 1968), 129 sq. et surtout 265-69 et Fig. 20.

22 Giovan Paolo Lomazzo, Trattato dell'arte de la Pittura (Milan, 1584), livre sept, chapitre xvir, Della Forma delle Muse, 596-98. On verra notamment à la 598 la référence suivante: "Alcuni altri hanno voluto che [...] Thalia (rappresenti la stella della) Luna per l'humore della quale la terra verdeggia ".

23 Francesco Patrizi da Cherso, Discorso della diversità de i Furori poetici all'illustre Signor Mariano Savello. Il s'agit d'une lettre adresséc de Padoue, le 12 janvier 1552. Ce texte a été réédité en appendice à l'édition critique du traité Della Poetica (Florence, 1969), 1, 447-62. On verra notamment les pages 450,452 el 459

24 Hess, ed., Die Künstlerbiographien, 13. 
âges de Vasari, il avait substitué sept "gouverneurs " qui sont les prototypes de génies artistiques et pour ainsi dire, de familles d'esprit. Ces sept gouverneurs sont les sept piliers sur lequel repose le Temple de la peinture. L'édifice est achevé et tout artiste à venir se situera nécessairement dans l'une des sept classes. Les sept gouverneurs qui soutiennent le Temple sont chacun l'incarnation de l'une des sept planètes. Il serait d'ailleurs plus exact de dire que c'est la planète qui est le gouverneur, l'artiste-type étant celui qui réalise le plus précisément le caractère de sa planète dominante. Ainsi Michel Ange est gouverné par Saturne, Titien par la lune, Léonard par le soleil. Cette association de l'artiste et de la planète gouvernante est opérée par ce que l'on pourrait appeler un horoscope objectif, c'est-à-dire les traits caractériels de l'artiste et les traits stylistiques de sa peinture, entendus comme expression graphologique du caractère de son auteur. Ce Temple esı universel : à chaque gouverneur et à chaque artiste correspondent un métal, un animal, un sage, un poc̀te, un artiste antique et l'une des sept parties de l'art. A Titien, par exemple, gouverné par la lune, correspondent l'argent, le boeuf, Aristote, l'Arioste, Aristide et la couleur. A l'artiste-type sont associés d'autres artistes secondaires qui réalisent l'idéal de façon moins parfaite. A Titien sont associés Giorgione, Véronèse, Tintoret et Peterzano, le maître du Caravage. Le Temple de la peinture apparaît ainsi comme une architecture symbolique, à la fois édifice mnémonique dont la fonction pédagogique est patente, édifice magique qui rassemble en soi tous les influx de l'univers et expose leur diversité dans la forme unifiante d'un Tempietto, apologie de l'Italie et mausolée. Le Temple possède toutes ces fonctions puisqu'il est un double de l'univers et une représentation des lois qui le gouvernent.

Du Temple de Lomazzo, Bellori a retenu la notion d'universalité que le Journal de Sçavans constatait avec tant de plaisir. Il a retenu aussi l'idée de l'édifice de mémoire et de mausolée: dans une lettre du 15 octobre 1667 à C. R. Dati, Bellori félicite l'antiquaire dont l'éloquence vient de rendre par son ouvrage sur les peintres antiques "les couleurs de Zeuxis, de Parrhasios, d'Apelle et des autres célèbres peintres qui avaient été effacés du Temple ${ }^{25}$. Il a retenu aussi et surtout la notion de gouverneur. Annibale Carrache, Caravage et le Dominiquin sont les "gouverneurs" de l'édifice de Bellori. L'analyse des vignettes gravées nous

25 " [...] con li colori dell'eloquenza non solo all'uditù ma alla vista e a gli occhi rappresenta li colori di \%eussi, di Parrasio, di A pelle ct de gli altricelebri Pittori cancellati dal Tempio " (Lettere di diversi a Carlo Roberto Dati, Florence, Bibl. Naz. Cent., Racc. Baldovinetti, Misc. 258, fasc. II, fol. 34). montrera dans quel sens précis il faut l'entendre. Chacun des trois peintres gouverne trois artistes et l'on peut aisément diviser les douze biographies en trois groupes: 1) Annibale suivi d'Augustin Carrache, D. Fontana, F. Barroci; 2) Caravage suivi de Rubens, Van Dyck, Duquesnoy; 3) Le Dominiquin suivi de Lanfranc, l'Algarde et Poussin.

Nous touchons ainsi à l'un des aspects les plus étonnants des Vite de Bellori, celui qui a provoqué peut-être le plus de perplexité : Caravage, dont Bellori rejette le naturalisme dans l'Idea, apparaît dans les Vite suivi de trois artistes flamands. La raison en est simple et Bellori la précise : les trois peintres ont suivi l'exemple de la peinture vénitienne. Caravage s'est donné Giorgione comme maître ${ }^{26}$. Rubens a suivi Titien, Véronèse et Tintoret $^{27}$. Van Dyck a pris le même chemin ${ }^{28}$. Tous trois furent animés d'une identique fureur de peindre. Tous trois ont manqué des mêmes parties de l'art : le dessin et la rigueur de la composition ${ }^{29}$. Quant à Duquesnoy qui a su représenter les chairs tendres, il a pris son "idée " de l'étude du Titien et $\mathrm{du}$ naturel et n'a pas traité d'histoire ${ }^{30}$. Bref, tous les quatre ont été animés de la même virtù naturaliste et sont d'abord des coloristes et des "vénitiens".

Ce type de rapprochement n'empêche pas Bellori de vouloir distinguer le mieux possible ce qui fait l'originalité de chaque artiste et il sait rendre à Rubens ce qui lui revient : sa culture, la vivacité de son dessin, sa capacité d'invention des grandes compositions $^{31}$. On a souvent remarqué que Bellori était capable de maîtriser son goût personnel pour reconnaître et apprécier par exemple l'originalité du Caravage ${ }^{32}$. Bellori est d'abord un lettré et un historien, soucieux de bâtir un modèle de la peinture parfaite et l'image d'un Temple Universel. Le "gouverneur" à la manière de Lomazzo est un moyen commode d'éviter les contraintes, les généralisations et les inévitables falsifications qui accompagnent la notion d'école, notion dont l'expérience contemporaine montrait les limites et que l'entreprise touffue de F. Scannelli n'invitait guère à employer ${ }^{33}$. Le milieu des amateurs romains s'était longuement interrogé sur ces problèmes de classification des oeuvres et des artistes. On connaît les classes stylistiques du marquis Giustiniani et les réflexions de Poussin sur

26 Bellori, Vile, 212-13.

27 Bellori, Vite, 241 et. 267

28 Bellori, Vite, 273.

29 Bellori, Vite, 230, 267 et 283.

30 Bellori, Vite, 299-3()0.

31 Bellori, Vite, 266-67.

32 Bellori, Vile, 229.

33 Irancesco Scannelli, Del Microcosmo della Pittura (Cesena, 1657). 
l'application aux sujets de peinture des modes harmoniques et poétiques de l'antiquité ${ }^{34}$.

Il est vraisemblable que Bellori trouva un modèle tout prêt dans le recueil poétique d'Antonio Bruni Le Tre Gratie publié à Rome en 1630. Bruni était l'un des poètes à succès de sa génération après le Cavalier Marin. Il était membre de l'académie des Humoristi et connaissait à peu près tout ce qui comptait à Rome et à la cour d'Urbain virI, Agostino Mascardi, Lucas Holstenius, Giulio Rospigliosi, Cassiano dal Pozzo, F. M. Suarez... Il connaissait aussi Ottavio Tronsarelli, celui-là même qui avait encouragé le talent poétique de Bellori. Il explique longuement au début du recueil le titre qui peut paraître nouveau et étrange au lecteur ${ }^{35}$. Son but est de classer les oeuvres poétiques d'une façon plus efficace et plus vraie. Bruni rappelle longuement que les Anciens ont attribué les diverses formes de la poésie aux neuf muses et que nombre d'auteurs ont publié leurs recueils sous le titre des muses. Pour Bruni cette division de la poésie est peu sûre et difficile à utiliser en raison surtout du mélange de genres différents à l'intérieur d'une même pièce poétique. C'est pourquoi il propose de diviser et classer la poésie en trois: Aglaia aura la poésie lyrique, amoureuse, son nom même l'invitant à chanter la Beauté; Thalia dont le nom signifie force et vigueur aura la poésie héroïque; Euphrosyne enfin dont le nom signifie le plaisir (diletto) aura la poésie sacrée et morale ${ }^{36}$.

On peut examiner le genre de pièces que Bruni classe sous le mode héroïque. Une première série est facile à deviner: il s'agit de tout ce qui se rapproche de l'épopée, par exemple ce poìme dédié à Alessandro Tassoni "l'invitant à chanter l'empire de Charlemagne en Italie ${ }^{37}$ ou cette autre pièce dédiée à Agostino Mascardi sur "l'Amour de la Patrie préféré à l'amour paternel ${ }^{38}$. Une seconde catégorie est constituée de tombeaux, réels ou imaginaires: le tombeau de Michel Ange, celui de Raphaël qui est une paraphrase du distique de Bembo ou le tombeau d'Hercule ${ }^{39}$. Troisième catégorie, les portraits héroïques, bustes des Grands

34 Il s'agit du célèbre essai envoyé sous forme de lettre par Vincenzio Giustiniani à Amayden. On le trouvera dans le recueil de Giovanni Bottari, Raccolta di lettere sulla pittura, scultura ed architeltura (Rome, 1768), vi, 247-53. Quant à Poussin, il s'agit de la lettre dite "sur les modes " adressée à Chantelou le 24 novembre 1647. On la trouvera dans Nicolas Poussin, Lettres et propos sur l'art (Paris, 1964), 121-25.

35 Antonio Bruni, Le Tre (iratie (Rome. 1630), 2.

36 Bruni, Le Tre Gratie, 19-20. L'attribut d'Euphrosyne sera: "il cerchio intrecciato di stelle".

37 Bruni, Le Tre Gratie, 258-361.

38 Bruni, Le Tre Gratie, 367 sq.

39 Bruni, Le Tre Gratie; Michel Ange se trouve page 295, Raphaël page 393, Hercule page 357 . du passé classique, Caton d'Utique, Cicéron, Enée, Néron, Mécène (un buste en cire!) ${ }^{40}$, et figures en pied des Grands contemporains, grands capitaines de préférence, le marquis Spinola, Filippe iv, Philibert de Savoie, Ranuccio Farnèse ${ }^{41}$. D'autres pièces tombent dans la catégorie de l'héroïque parce qu'elles peuvent s'apparenter au style fier et terrible : témoin ce poème sur un phénix peint par le Caravage ${ }^{42}$.

On voit la coincidence de cette classification avec les sujets-qu'ils soient de portraits ou d'histoire-des artistes du second groupe de Bellori. On voit aussi la parenté du mode héroïque de Bruni avec la fierté stylistique et la véhémence du Caravage et des artistes qui lui sont associés. On peut aussi noter que Bellori, pour donner plus de cohésion à son groupe "hérö̈que ", a insisté sur leur parenté caractérielle: tous ont voulu mener grand train, ont aimé le luxe, les vêtements somptueux, la compagnie des grands, les honneurs et les faveurs qui viennent des cours ${ }^{43}$. Bruni chante le don princier d'une chaîne d'or qu'il a reçue ${ }^{44}$. Dant nobis praemia reges dit la vignette du Rubens de Bellori (Fig. 45) ${ }^{45}$.

On peut faire les mêmes observations pour les poésies sacrées et morales d'Euphrosyne qui regroupent à la fois des vanités, des sujets de méditation (une Madeleine, Phaeton, l'Age d'or) et des sujets d'histoire sainte (Annonciation, Nativité) ${ }^{46}$.

L'étude des pièces appartenant à la première Grâce confirme qu'il s'agit d'une classification "modale". Aglaia regroupe tous les types de poésie lyrique, des portraits de femmes et l'évocation de certains tableaux: Iole riant de Ludovic Carrache, la Cléopâtre du Guide, un Adonis de Guiseppe d'Arpino, un Amour de Baglione ${ }^{47}$. Ce n'est pas, à l'évidence, l'unité stylistique qui pousse Bruni à regrouper ces tableaux sous l'autorité d'une même Grâce mais bien plutôt le ton, l'harmonie générale, l'humeur du sujet, le mode pour reprendre le terme employé par Poussin.

\section{I.ES VIGNETTES}

Chacune des douze biographies est précédée d'un portrait gravé de l'artiste et d'une vignette allégorique. Dès la parution de l'ouvrage on a remarqué la qualité de ces gravures et l'on peut imaginer l'importance que Bellori y attachait. La question

40) Brumi, Le Tre Gratie, 363, 364 et 365.

41 Bruni, Le Tre Gratie, 347, 352 notamment.

42 Bruni, Le Tre Gratie, 295.

43 Bellori, Vile, 264-65 et 283, par exemple.

44 Brumi, Le Tre Gratie, 380.

45 Bellori, Vile, 239

46 Bruni, Le Tre Gratie, 405 sq.

47 Bruni, Le Tre Gratie, 97, 137 et 173. 
s'est évidemment posée de savoir si ces vignettes n'étaient pas détentrices du secret du programme iconographique des Vite. La réponse fut toujours négative. Ce qui semble compliquer les choses c'est la constatation que deux ordres différents d'allégorisation paraissent avoir été choisis par Bellori : un ordre idéal de définition de la peinture, poésie muette, choix et discernement, activité intellectuelle; puis un ordre de définition des parties de la peinture, la géométrie, la praxis, l'étude de la nature, la lumière et l'ombre. Bref, le plus grand désordre semble régner et la plus grande confusion. Caravage reçoit le motto Praxis, généralement attaché - et par Bellori lui-même en 1664-aux maniéristes (Fig. 44). Lanfranc par contre est associé à Natura et Poussin a pour attribut Lumen et Umbra (Figs 49 et 51$)^{48}$.

Si nous observons la série des treize vignettes en suivant l'ordre de l'édition (Figs 39 à 51) nous constatons que sur treize gravures, une seule est dépourvue de motto et présente une mise en scène différente. Il s'agit de la vignette qui accompagne la vie d'Annibale Carrache et qui représente la Renommée volant et sonnant de la trompette (Fig. 40). Par contre la représentation de l'Idea qui précède la préface, tant par son style que par sa stricte mise en scène dans une architecture héroïque, appartient très clairement à la même série que les onze allégories titrées (Fig. 39). Il devient facile de rétablir le programme original: l'allégorie de l'Idea était destinée à Annibale Carrache. En fait nous allons voir que son iconographie - les pointes du compas dressées vers le haut-l'identifie à la Théoria telle que définie par Ripa. Elle s'oppose alors tout naturellement à l'allégorie du second gouverneur, Caravage: la Praxis, symétrique de la théorie et qui mesure le sol. Au troisième gouverneur, le Dominiquin, est associé le Concept et l'Imagination (Fig. 48).

On voit - et c'est une première constatationque l'édition de la Conférence de l'Idea de 1664 n'avait sans doute pas été prévue dans le programme originel ${ }^{49}$. Il est facile d'imaginer la suite : au moment de publier l'ouvrage, Bellori décide de le faire précéder de la conférence prononcée huit ans plus tôt. Malheureusement la décision vient tard et toutes les vignettes du programme ont déjà été gravées à l'académie de France sous la surveil-

48 Sur cette apparente confusion du programme on lira les remarques de $G$. Previtali dans l'introduction à l'édition des Vite (Turin, 1976), notamment aux xliv-l.

49 On pourrait soutenir que l'édition de la conférence avait été prévue mais sans vignette. Cela ne serait guère vraisemblable. Il suffit d'ouvrir l'ouvrage pour constater avec quel soin minutieux ont été réalisées la gravure dédicatoire à Colbert et la vignette qui accompagne la dédicace proprement dite. lance de Charles Errard, ami de Bellori. Il faut une vignette supplémentaire. Le graveur réalise rapidement l'image de la Renommée (Fig. 40). De cette rapidité d'exécution témoignent la simplicité de la composition, l'importance accordée aux zones d'exécution facile (ciel, nuages), à la grande part laissée aux blancs et au peu de soin dans le travail des détails (drapés surtout, palme, ailes). Il suffit pour bien saisir ce point de comparer cette vignette avec l'Idea (Fig. 39) ou la Muta poesis (Fig. 41). Au moment d'imprimer se produit l'une de ces erreurs d'éditeur très fréquentes au $\mathrm{xvII}^{\mathrm{e}}$ siècle : à la conférence de l'Idea est associée la vignette du même nom. Du même coup, le programme théorique de Bellori qui, pour tout lecteur du xvir ${ }^{\mathrm{e}}$ siècle, était visuellement évident par la symétrie bien connue du couple Théoria-Praxis (Figs 39 et 44), ce programme devient inintelligible dès que l'on déplace l'un de ses piliers.

Pour un amateur du xvir siècle, Theoria et Praxis sont les divisions traditionnelles de la peinture. Tous les traités depuis Alberti reprennent cette division qui permet de définir la peinture non seulement comme art mécanique mais aussi comme science spéculative. L'idée qui revient sans cesse est très simple: la théorie, fondée pour l'essentiel sur la mathématique, donne à la pratique picturale les bases fermes, assurées, universelles qui font de la peinture une science véritable et lui donnent sa certitude. Lomazzo exprime ainsi cette idée au chapitre 2 du premier livre du Trattato :

La pcinture se divise en théorique et pratique. La théorique donne des préceptes généraux que doit observer celui qui veut devenir excellent et célèbre dans cet art. La pratique donne des règles de prudence et de jugement et enseigne comment mettre en oeuvre ce qui s'est dit et imaginé de façon générale ${ }^{50}$.

Léonard avait exprimé à peu près la même idée de façon plus sentie:

Ceux qui s'amourachent de la pratique sans la science sont comme des pilotes à bord d'un navire sans gouvernail ni boussole et qui ne savent jamais avec certitude où ils vont.

La pratique doit toujours s'édifier sur la bonne théorique; la perspective en est le guide et le chemin et sans elle on ne fait rien de bien en peinture ${ }^{51}$.

50 " Dividesi la pittura in theorica, e prattica. La theorica da precetti generali, che deve osservare ciascuno che vuole divenire eccellente, e famoso in quest'arte. La prattica da regole di prudenza, e giudicio, insegnando come si hà da mettere in opera quello che si è detto, e imaginato generalmente "(Lomazzo, Trattato, 28)

51 "Quelli che s'inamorano di praticha sanza scientia sono com'e li nochieri ch'entran in naviglio sanza timone, ò bussola che mai hanno certezza dove si vadano. Sempre la praticha debb'esser'edifficata sopra la bona theoricha della quale la prespettiva è guida è porta e'sanza questa nulla si fa 
De cette idée qui était devenue au cours du $\mathrm{xvI}^{\mathrm{e}}$ siècle un lieu commun des trattatistes, le graveur Pietro Testa avait, vers 1638 donné une représentation impressionnante. Il s'agit du célèbre Liceo della Pittura qui, suivant l'exemple formel de l'Ecole d'Athènes, reproduit le modèle de la peinture idéale, et le programme de son enseignement dans l'académie parfaite (Fig. 52) ${ }^{52}$.

On sait que Testa travaillait à Rome, notamment pour Cassiano dal Pozzo, qu'il fréquentait le milieu classicisant et il ne fait aucun doute que Bellori eut connaissance de son oeuvre et de ses recherches ${ }^{53}$.

Une inscription située à la gauche de la composition donne les raisons du programme iconographique :

La Théorique, en soi, a les mains liées et la Pratique dans sa liberté est en soi aveugle. Mais celui qui cngagé dès ses jeunes années dans l'étude de la peinturc apprend ce qui est bon des grands maîtres, puis s'avance à imiter de soi-même les objets de la nature, cntre dans lc I.ycée de Pallas; il y retrouve et y comprend les arts de la Mathématique, il unit la Théorie à la Pratiquc et les débarrassant de leurs défauts respectifs par l'heurcuse union de l'intelligence et de l'usage, il se fait un nom glorieux et accroît dans le monde les récompenses de la vertu ${ }^{54}$.

La composition est ordonnée par une scénographie assez simple : au fond, autour de la statue de Minerve, des prêtres de divers cultes, un obélisque, un sacrifice; sur la scène, deux groupes principaux : à gauche, la vie contemplative dominée par les statues de la vérité et de la mathématique portant un compas aux pointes levées. Devant la mathématique des personnages figurent l'arithmétique, la géométrie, l'astronomie, la musique (Fig. 54). A droite, dominés par les statues de l'éthique et de la félicité publique, figurant la vie active, se trouvent l'art oratoire, l'art politique, l'art militaire, l'économie familiale (Fig. 54). Au premier plan mais hors de la scène, la théorie et la

bene ne casi de pittura "(Codex Urbinas Latinus, 1270, ed. A. Philip McMahon [Princeton, 1956], II, fac-similé, fol. $39 v)$.

52 A. Bartsch, Le peintre graveur (1870), xx, 223.

53 Sur P. Testa on lira, outre l'ouvrage d'F. Cropper, The Ideal of Painting (Princeton. 1984), la biographie écritc par Passeri et publiée par Hess, ed., Die Künstlerbiographien, I, 18288.

54 "La Theorica è per se stessa di leggaıni avvinta c la Prattica nella sua libertà è per stessa cieca; ma chi in età di freschi anni ne gli studij della pittura il buono di gran maestri apprende; e poi avanzandosi ad imitare da se gli ogetti della natura, entra nell'dotto Liceo di Pallade, c vi ritrova et intende le arti della Mathematica unisce egli la l'heorica alla Pratica; e spogliando le de loro difelti con felice acoppiamento dall'intelligenza e dall'uso a sc acquista gloria di nome e al mondo accresce pregi di virıù ". Les ınêmes idćcs se retrouvent encore chez Passeri. On verra notamment les deux projets qu'il avait écrits pour le Proemio; cf. Hess, cd., Die Künstlerbiographien. I, 9 et 16 où Passcri fait dircctement référence au Liceo. pratique. Testa en a emprunté la représentation à Fulvio Mariotelli dans l'édition de 1625 de l'Iconologia de Ripa (Fig. 55). C'est une jeune femme qui personnifie la théorie. Elle porte sur la tête un compas dont les pointes sont tournées vers le haut. A l'intérieur de l'arc de cercle décrit par l'ouverture du compas, est figuré un théta. A ses pieds des livres qu'elle ne peut ouvrir ni consulter parce que ses mains sont enchaînées (Fig. 53). La Pratique est une vieille femme, aveugle, tenant à la main un maalstok avec lequel elle tente de guider sa marche. Elle avance sur un sol parsemé de rochers. A ses côtés, un singe mendiant - allusion aux naturalistes qui copient ce que le hasard leur offre. Au-dessus de sa tête est figurée la lettre pi (Fig. 54) ${ }^{55}$. Cette figure allégorique, comme la précédente, vient de l'imagination de Fulvio Mariotelli et de l'édition de 1625 de Ripa (Fig. 56).

Au centre de la composition se trouvent les trois degrés par lesquels l'étudiant accède au Lycée: l'imitation est suivie de l'observation, suivie ellemême de la perfection, c'est-à-dire la raison. La figure du Jugement accueille le jeune peintre et désigne le personnage de la Peinture qui est conduite par l'Optique, portant une planche pleine de figures géométriques. Il devient ainsi facile de rétablir le premier groupe d'artistes du programme de Bellori, artistes rassemblés autour d'Annibale Carrache, le gouverneur "spéculatif " (Figs 39, 41, 42 et 43):

$\begin{array}{ll}\text { Idea } & \text { Annibale Carrache } \\ \text { Muta poesis } & \text { Augustin Carrache } \\ \text { Geometria } & \text { D. Fontana } \\ \text { Studio vigilanti } & \text { Le Baroche }\end{array}$

On voit que Bellori a conservé les articulations principales du programme d'éducation représenté par Testa, hérité de la tradition des trattatistes, mais certaines modifications méritent toutefois examen. Chez Bellori l'image de l'I dea est suivie de la Muta poesis (Fig. 41), puis de la Geométria (Fig. 42), enfin de l'image de l'étude (Fig. 43). A l'intérieur du Temple de la Peinture, nous sommes du côté de la vie contemplative, du côté de la Théorique et du spéculatif. La Muta poesis (Fig. 41) fait le geste du silence; elle est vêtue de la robe étoilée d'Uranie et porte une couronne de laurier, conformément à l'image donnéc par Ripa (Fig. 55). La poésie en effet n'est pas autre chose que l'expression des choses divines provoquée dans l'esprit par la fureur et la grâce céleste, et la robe étoilée signifie la divinité car on dit que les poètes viennent du ciel ${ }^{56}$. Ripa toutefois avait prévu que la

jう On lira la description détaillée de la composition dans E. Cropper, dans Cropper, The Ideal of Painting, 65-95.

56 "Poesia, secondo Platone, non c altro, ch'cspressione di 
figure serait accompagnée d'instruments de musique. Bellori lui a donné (s'il est bien l'auteur des détails du programme iconographique) un livre qu'elle tient ouvert de la main gauche. On retrouve ce livre ouvert dans l'image de l'étude et on le retrouve aussi à l'état de planche de figures dans la Géometria (Fig. 42). C'est dans la vie d'Augustin Carrache que Bellori donne l'explication de la présence de ces livres. "Poussé dès l'enfance par un très vif amour du savoir, il s'attacha aux sciences et aux arts. Il apprit les mathématiques, la philosophie puis passa à la rhétorique, à la poésie, à la musique et à tous les autres arts libéraux, $[\ldots] \|^{57}$.

Il n'est pas trop utile d'expliquer davantage la vignette qui représente l'étude et la vigilance (Fig. 43), si ce n'est pour préciser que cette image s'applique d'abord à la partie spéculative de l'art, ce qui est le sujet du premier groupe et non en particulier au personnage historique du Baroche ${ }^{58}$. On pourrait dire la même chose de la Geométria qui préside à la vie de Fontana (Fig. 42). Cette image mérite examen attentif, car la geométrie, selon Ripa, est représentée par une femme qui tient un équerre et un compas ${ }^{59}$. La vignette de Bellori est plus complexe : c'est en fait une adaptation de la Mathématique de Ripa (Fig. 58). La Mathématique se représente comme une femme grave et d'âge mûr, ayant deux ailes sur la tête. Elle tient un compas et trace un cercle sur une planche couverte de figures géométriques que tient un enfant. Son

cose divine eccitate nella mente da furore et gratia celeste [...]. La veste con le stelle, significa la divinità, per conformità di quello, che dissero i Poeti haver origine dal Cielo "(C. Ripa, Iconologia [Rome, 1603], 406-4()7). Il semble que les graveurs aient utilisé au moins trois ćditions de l'Iconologia, celle de 1603, celle de 1625 et celle de 1645.

Contraircment à Testa qui place tout le spéculatif sous le gouvernement de la Mathématique, Bellori semble avoir choici la Poésie. Certains traits de la vignette intitulée Idea appartiennent au Furor poetico défini par Ripa (Fig. 20; 1603), 178, notamment qu'elle dessine (ou peigne) et qu'elle ait les yeux tournés vers le ciel, l'inspiration poétique se représente ainsi : "Giovane vivace, e rubicondo con l'ali alla testa coronato di lauro, c cinto d'edera stando in atto di scrivere : ma con la faccia rivolta a Cielo ". Dans l'édition de 1645, publić à Venise, l'Idea est ainsi représentée: "Si dipinge nucla per essera spogliata da ogni passione corporca".

57 " (Agostino Carracci, il quale) dalla pucrizia acceso da un ardentissimo amore di sapere, si pose nel giro delle scicnze et clelle arti. Attese alle mathematiche ed alla filosofia, c da queste si rivolse alla rettorica, alla poesia, alla musica ed ad ogn'altra facoltà liberale [...]" (Bellori, Vite, 115).

58 Ripa, Iconologia, 478. Le Studio est ainsi représenté : "Ln giovane di volto pallido, vestito d'habito modesto sarà à sedere, con la sinistra mano terrà un libro aperto, nel quale miri attentamente, con la clestra una penna da scrivere, e gli sarà à canto un lume accesso, e un gallo "; on voit que le graveur de Bellori a idéalisé et, pour ainsi dire, archéologisé la définition de Ripa. Le coq est attribut de la vigilance. 59 Ripa, Iconologia, 183. vêtement porte une bordure ornée de triangles et d'autres figures géométriques. A ses pieds se trouve une pyramide. Nous retrouvons exactement les mêmes traits dans la vignette de Bellori si ce n'est que le personnage féminin s'est transformé en vieillard saturnien et qu'un feu occupe l'arrière plan de la composition. Qu'il s'agisse bien du même concept, cela est certain : l'artiste qui a travaillé pour Bellori s'est amusé à reproduire sur le vêtement la frise de triangles et les figures géométriques dont parle Ripa ${ }^{60}$. Le feu qui se trouve au fond de la composition ne peut dans ce contexte signifier qu'une seule chose : le désir de savoir. Ce désir est, chez Ripa, l'un des attributs de l'intellect (Fig. 59). Le feu que le personnage porte sur la tête est " né de la capacité et de la vertu intellective qui aspire toujours aux choses élevées et divines " à condition que ce désir de savoir ne se laisse pas dévier par les sens qui n'obéissent guère volontiers à l'intellect et le poussent vers le bas. Revenons un instant à l'Idea de Bellori (Fig. 39). Elle peint, les yeux tournés vers le ciel; entre les pointes du compas on voit un feu qui surgit de sa chevelure, avec la même signification ${ }^{61}$. On peut d'ailleurs noter en passant l'évolution qui s'est produite depuis que Testa en 1638 a représenté la Mathématique ailée à

60 "Donna di mez'età, vestita di velo bianco è trasparenticon l'ali alla testa, le treccie siano distese giù per le spalle, con un compasso nella destra inano, mostri di misurare una tavola segnata d'alcune figure, e numeri, e sostentata da un fanciullo al quale ella mostri di parlare insegnandoli [...] e nel lembo clella veste sia un fregio intessuto di figure Mathematiche $[\ldots]$

"Il compasso è l'istromento proprio, e proportionato di questa professione, e inostra che ella di tutte le cose dà la proportione, la regola, e la misura.

"Stà in atto di tirare il circolo, perche se bene la Mathematica è speculativa scienza, denominandola dal suo più vero, e nobil fine, nondimeno ancora l'uso, è fine, se non della scienza almeno di chi la possiede, essendo necessario dopo l'acquisto dell'habito d'essa per giovamento dell'altri manifestarla in qualche modo, e di qui sono nate l'inventioni di musiche di prospettiva, di Architettura, di Geometria, d'Aritmetica, e d'altre professioni [...]

"Il compasso alla Mathematica, e il fregio di triangoli, e d'altre figure intorno alla veste, mostra che come sono nel lembo i fregi d'ornamento e di fortezza, cosi nelle prove Mathematiche queste istesse sono principij e fondamenti " (Ripa, Iconologia, 307-309).

On notera que l'illustration de l'ćdition de Padoue de 1625. nc montre quiun vêtement uni.

61 l'Idea de l'édition de 1645, publiée à Venisc, 362-65, a un feu au sommet de la tête, signifiant le bien. Ce feu est toutefois de puis l'édition de 16() 3 un attribut clair de l'intellect. " la fiamma è il naturale desiderio di sapere, nato dalla capacità della virtù intellettiva, la quale sempre aspira alle cose alte, e divine, se da sensi che mal volontieri l'obediscono alla consideratione di cose terrene, e basse non si lascia sviare"(Ripa, Iconologia, 329). Que le feu soit représcnté deux fois dans les vignettes du premier groupe d'artistes n'a rien d'étonnant puisqu'il s'agit du domaine du spéculatif qui s'oppose au sensible des artistes groupés aucour de la Praxis. 
la manière de Ripa: en 1672, dans le milieu classicisant français et romain, l'artiste recule devant l'utilisation d'un symbole sans vraisemblance. Ne pouvant imaginer une Mathématique à la tête ailée, il va employer le feu qui signifie la même chose, et le porteur des attributs symboliques devient une sorte d'Archimède mélancolique ${ }^{62}$.

\section{I.A PRAXIS}

Le second groupe des vignettes allégoriques est commandé par la Praxis, placée en tête de la vie du Caravage (Fig. 44). La séquence est la suivante (Figs 44 à 47):

\section{Praxis \\ Dant nobis praemia reges \\ Imitatio sapiens \\ Calamo ligantur eodem}

Caravage
Rubens
Van Dyck
Duquesnoy

Il est assez facile de comprendre la signification des allégories. Le premier point - et le plus important - est l'explication de la Praxis associée au Caravage. Bellori dans la Conférence de 1664 avait associé la pratique aux maniéristes. Il n'innovait d'ailleurs pas en cela. De son côté Testa avait associé la pratique à l'imitation naturaliste, à l'observation de la nature et c'est cette association que Bellori va reprendre à son compte. Il ne semble pas qu'il se soit renié pour autant. Dans la Conférence de l'Idea de 1664, il avait condamné ensemble naturalistes et maniéristes.

C'est pourquoi ceux qui sans connaitre la vérité lont tout de pratique, représentent des ombres plutôt que des figures. lit les autres ne sont guère différents qui placent avant tout le génic particulier, copient les idées d'autrui et font ainsi de leurs oeurres des bâtards et non des enfants légitimes de la nature, comme s'ils avaient juré fidélité aux pinceaux de lcurs maîtres. A ce mal s'ajoute - par manque de génic-lcur incapacité à choisir le meilleur. On les voit plutôt préférer les défauts de leurs précepteurs et se former l'idcée du pire. Au contrairc ceux qui se glorifient du nom de naturalistes ne se

62 La pyramicle de la vignelle bellorienne est assez curieuse. Elle vient sans doute de la Mathématique de Ripa mais elle peut venir aussi (et ce n'est pas contradictoirc) de l'article "Gloria de'Prencipi nella medaglia d'Adriano", dans Iconologia, 190-91 : "Et similmente gl'antichi mettevano le piramidi per simbolo della gloria, che pero s'alzarono le grandi, e magnifiche piramidi dell'ligitto [...] ". La vignette ćtant appliquéc à Fontana, le restaurateur des obélisques, le renovateur de la science égyptienne et l'architecte de la gloire des Pontifes, cette signification est possible. La première phrase de la biographie de Fontana par Bellori la rend probable: "D. Fontana fu architetto molto celebre per l'crezzione de gli obelischi, onde acquistossi eterna fama: percioché la novità e la difficoltà di tale impresa, (lopo mille e duecento anni senza essempio ed insegnamento, e da gli ligizii stessi autori di si stupende moli riputata grandissima, faceva trepidare li moderni architetti ed aveva ritardato la magnificenza de'sommi pontefici in farle risorgere dalle rovine di Roma "(Bellori, Vite, 151). proposent à l'intellect aucune idée. Ils copient les défauts des corps et se font une habitude de la difformité et des erreurs, ne jurant que par le modèle, leur seul maître ${ }^{63}$.

Le programme du second groupe est de fait plus complexe qu'il ne semble à prime abord. Nous retrouvons l'image du premio, associée à Rubens, c'est-à-dire la récompense que l'artiste reçoit des princes (Fig. 45). Elle vient telle quelle de Ripa (Fig. 60) ${ }^{64}$. Limage du tuteur, associée à Duquesnoy (Fig. 47) est bien évidemment l'image du bon maître qui surveille la croissance de la jeune plante et lui offre l'appui sans lequel elle ne pourra se développer ${ }^{65}$. Nous pouvons imaginer que la vignette du premio est en quelque manière associée au pôle de la vie active défini par Testa. L'image du tuteur convient tout aussi bien aux naturalistes qu'aux maniéristes et l'examen des deux autres vignettes confirme que Bellori a voulu faire coup double. L'imitation n'est pas représentée par le singe mendiant comme chez Testa mais par une image de la vertu dc Prudence (venue sans doute du traité de Lomazzo), qui domine le singe (Fig. $46)^{66}$. Ce n'est donc pas la représentation de l'imitation directe par le peintre des choses de la nature mais d'une imitation raisonnée qui sait choisir le meilleur et laisser les défauts. La Praxis, ellemême, est construitc de façon plus complexe qu'il n'y paraît (Fig. 44). Mariotelli l'avait représentée

6.3 "I aonde quelli che senza conoscere la verità il tutto muovono con la pratica, fingono larve invece di figure; né dissimili gli altri sono, che pigliano in prestanza l'ingegno e copiano l'idee altrui, lanno l'opere non figliuole, ma bastarde della natura, e pare abbiano giurato nelle pennellate de'loro maestri. Al qual male si aggiunge che, per l'inopia dell'ingegno, non sapendo essi eleggere le parti migliori, scelgano i difetti de'loro precettori e si formano l'idea del peggiore. Al contrario quelli, che se gloriano del nome di naturalisti, non si propongono nella mente idea alcuna; copiano i difetti de'corpi c si assuefanno alla bruttezza ed a gli errori, giurando anchi'essi nel modello come loro preccttore [...]" (Bellori, Vite, 21-22).

64 "Huorno vestito di bianco, cinto d'un velo d'oro, tenendo nclla clestra mano una palma con un ramo di quercia, e nclla sinistra corone, e ghirlande "(Ripa, Iconologia, 41112).

65 Ripa, Iconologia, 28. Sous l'article "Arte ", on trouve l'explication de la vignette: "Donna vestita di verde, nella mano dritta tiene un pennello, e un scarpello, c con la sinistra un palo fitto in terra, al quale vi sia legata una pianta ancora novella e tencra. Il pennello e lo scarpello significano l'imitatione della natura, che particolarmente si vede espressa nel dipingere, e nello scolpirc [...] e perche in alcune altre non imita, ma supplisce à i difetti d'essa, come nell'Agricoltura particulare, pero vi s'aggiunge il palo fitto in terra, quale con la sua drittura fa, che per vigor dell'arte cresca il torto, e tenero arboscellon".

66 Chez Ripa, Iconologia, 223, l'imitation est ainsi représentće : "Donna che nella mano destra, tienc un inazzo di pennelli, nclla sinistra una maschera, e à picdi una scimia [...]. La maschera, c la scimia, ci dimostrano l'imitatione dell'attioni humane; questa per essere animale, atto per imitare l'huomo co'suoi gesti [...]". 
comme une vieille femme pauvre, amaigrie, marchant avec difficulté (Fig. 56). Le graveur de Bellori a choisi une mise en scène différente: une femme robuste, assise, tenant le compas et le fil à plomb et construite approximativement sur le schéma de la lettre pi. Selon Ripa,

Ce terme Prattica [...] signifie une chose relative opposéc à la Théorique [...]. On peut la représenter sous les traits d'une vieille femme la tête et les mains tournées vers la terre, vêtue comme une servante. [...] Elle tient un grand compas ouvert dont l'une des pointes est plantée dans le sol. Elle s'appuic d'une main sur le dit compas et de l'autre sur une règle de façon à ce que l'autre pointe du compas ouvert touche le sommet de la règle, formant ainsi la lettre par laquelle les grecs désignaient la pratique comme ils désignaient la théorie par le $\theta^{6 i}$.

L'artiste a choisi un modèle quelque peu différent: par sa pose, sa corpulence, sa physionomie lourde, sa musculature, sa coiffe, son drapé, sa chaise massive, la disposition et la taille même de ses pieds, la Praxis trouve sa source dans la Sybille de Cumes du plafond de la Sixtine. Par cette évocation de Michel Ange, maniéristes et naturalistes se trouvaient associés. Michel Ange ne jurait-il pas par la nature seule? N'avait-il pas produit une postérité de sophistes? Charles Errard, directeur de l'académie de France à Rome, était grand ami de Bellori. Il était aussi grand ami de Fréart de Chambray dont il a laissé un portrait fin et spirituel. On connaît les attaques que Fréart avait menées contre Michel Ange dans son Idée de la Perfection de la Peinture de 1662. Si Michel Ange avait été jugé par un jury composé des peintres antiques " $[\ldots]$ l'impertinence de son esprit en ce qui concerne l'invention et ses Idées cérébrines qui ne formaient que des expressions vilaines et ridicules l'eussent toujours rendu incapable d'être admis au rang des peintres [...] ${ }^{68}$.

Michel Ange ne possédait pas la Vérité de l'Histoire, ni la Convenance des Figures à leur sujet et au lieu où elles sont peintes. Il n'a aucune partie du Costume. Il est, pour ainsi dire, dépourvu de jugement ${ }^{69}$. Nous avons vu que Bellori reproche aux naturalistes leur manque de dessin et d'idée. Les reproches qu'il fait à Rubens sont les mêmes que depuis l'Arétin on fait à Michel Ange :

67 "Questa voce P'rattica suona [...] per significare cosa relativa e opposta alla Teorica. [...] si potrà far la Prattica vecchia con la testa e mani verso la terra, vestita servilmente [...] con un compasso grande aperto e con una punta fitta in terra, appoggiandosi con l'una delle mani sopra il detto compasso; con l'altra sopra un regolo, in modo che una punta del compasso aperto tocchi la sommità del regolo. per rappresentare insieme la lettera greca $\pi$ con la quale essi solevano significare la Prattica, come con la $\theta$ Teorica [...]" (C. Ripa, Iconologia [Padoue, 1625], 522).

$68 \mathrm{R}$. Fréart de Chambray, Idée de la perfection de la peinture (Paris, 1662), 70.

69 Fréart de Chambray, Idée de la perfection, 75
"Il conforma ses figures à une idée de visages et de barbes sans variété, tous semblables et plutôt vulgaires ${ }^{70}$. Le même reproche d'uniformité, il l'applique, comme on sait, à la manière du Caravage, l'autre Michel Ange, incapable de sortir de la cave, indigent d'invention et de dessin, privé de décorum, sans art et qui colorait toutes ses figures selon un seul éclairage et sur un seul plan, sans dégradé ${ }^{71}$.

\section{AGLAIA}

Aglaia, la troisième Grâce, appartient à la zone solaire proprement dite, celle de la Nature et de la Raison qui transcende l'observation des simples choses naturelles et unit l'observation à l'Idée. Qu'est-ce à dire? Le Dominiquin qui est le gouverneur du peintre apollinien eut comme premier talent d'exprimer les passions, de dessiner les esprits et de colorer la vie ${ }^{72}$. On connaît le célèbre contresens de Bellori, analysé par Panofsky.

Platon [...] dit que l'idée est unc parfaite connaissance dc la chose, connaissance issue de l'observation de la nature. Quintilien nous enseigne que toutes les choses amenées à la perfection par l'art et le génie humains ont leur principe dans la nature même d'où vient la véritable idée $e^{7.3}$.

Les vignettes allégoriques du troisième groupe d'artistes sont un commentaire de cette définition de l'idée. La séquence est la suivante (Figs 48 à 51 ) :

$\begin{array}{ll}\text { Conceptus-Imaginatio } & \text { Le Dominiquin } \\ \text { Natura } & \text { Lanfranc } \\ \text { Tres Sorores } & \text { L'Algarde } \\ \text { Lumen et Umbra } & \text { Poussin }\end{array}$

Il est facile de deviner que les artistes de ce groupe unissent les qualités des artistes des deux premiers groupes; ils unissent en eux Theoria et Praxis. Il serait trop aisé de commettre à notre tour un contresens : le Dominiquin de Bellori n'est en aucune façon un amalgame monstrueux, un peintre parfait à la manière de Lomazzo, issu de la chirurgie qui unit Michel Ange dessinateur et Titien coloriste ou Raphaël dessinateur et Corrège coloriste. Afin que nul ne commette ce contresens,

70 " Accommodo le sue figure ad una idea di volti e di barbc senza varietà e non dissimili fra loro e più tosto vulgari" (Bellori, Vite, 267).

71 Bellori, Vite, 218.

72 "Con la viva efficacia di esprimere gli affetti che fu sua propria [...] a lui toccò la gloria maggiore di linear gli animi e di colorir la vita "(Bcllori, Vite, 306).

73 " [...] Volendo Platone che l'idca sia una perfetta cognizione della cosa cominciata su la natura. Quintiliano c'instruisce, come tutte le cose perfezzionate dall'arte e dall'ingegno umano hanno principio dalla natura istessa, da cui deriva la vera idea "(Bellori, Vite, 21). Pour l'analyse de Panofsky on verra Idea, 105-109 et la discussion d'F. Cropper, The Ideal of Painting. 
Bellori cite, à la fin de la biographie du Dominiquin, une lettre du peintre à Francesco Angeloni, l'antiquaire - et parent de Bellori-où est dénoncée l'erreur de Lomazzo $^{74}$. Si le peintre parfait de Bellori est éduqué aux sciences et aux lettres tout autant qu'aux techniques, il se définit d'abord par sa capacité à représenter les actions hu maines dans l'Histoire, la Fable ou l'Allégorie, à les représenter dans la Nature, dans la lumière solaire.

En fait, la définition de l'idée par Bellori est d'abord déterminée par ceci: la peinture représente les actions, c'est-à-dire les passions qui conviennent aux actions, ce qui ne limite pas le travail à ce que l'on appelle l'expression mais s'étend à la définition même de l'histoire décrite et à sa mise en scène. Dans l'Idea de 1664 Bellori avait très clairement précisé sa pensée.

[...] la peinture étant une représcntation des actions humaines, le peintre doit avoir présent à l'esprit le modèle des passions qui conviennent aux actions, tout comme le poète garde l'idée du colérique, du timide, du triste, du joyeux et du rire, des pleurs, de la crainte et de l'audace. Ces passions doivent d'autant plus rester imprimées dans l'esprit de l'artiste -à l'aide de la contemplation continue de la nature - qu'il lui est impossible de les dessiner à la main d'après naturc s'il nc les a auparavant formées dans l'imagination. Pour ce faire la plus grande attention est indispensable: on ne voit jamais les mouvements de l'âme que fugitifs et soudains ${ }^{75}$

Ft Bellori poursuit en énonçant ce que l'on peut nommer le paradoxe du naturaliste, déjà énoncé par G. Mancini au début du siècle.

C'est pourquoi quand le peintre ou le sculpteur entreprend de représenter les opérations de l'âme issues des passions, il ne peut les observer dans le modèle qui se trouve en face de lui car le modèle est vide d'expression: il languit, l'esprit et les membres figés, immobilisé par une volonté ćtrangère. Il est donc nécessaire de se former une imagc à partir de la nature, d'observer les expressions humaincs, d'harmoniser les mouvements du corps aux mouvemonts de l'âme afin que les uns dépendent des autres et les autres des $u_{n}{ }^{76}$.

74 Bellori, Vite, 371-72

75 "Essendo la pittura rappresentazione d'umana azzione, deve insieme il pittore rittenere nella mente gli essempi de gli affetti, che (adono sotto esse azzioni, nel modo (he'l poeta conserva l'idea dell'iracondo, del timido, del mesto, del lieto, e cosi del riso e del pianto, del timore e del ardire. Li quali motti deono molto più restare impressi nell'animo dell'artefice con la continua contemplazione della natura, essendo impossibile ch'egli li ritragga con la inano dal naturale, se prima non li averà formati nella fantasia; ed a questo è necessaria grandissima attenzione; poiche mai si veggono li moti dell'anima, se non per transito e per alcuni subiti momenti "(Bellori, Vite, 20).

76 "Sichè intraprendendo il pittorc e lo scultore ad imitare le operazioni dell'animo, che derivano dalle passioni, non puo vederle clal modello che si pone avanti, non ritenendo esso alcun alfetto che anzi languisce con lo spirito e con le membra nell'atto in cui si volge, e si ferma ad arbitrio altrui. F. però neccssario formarsene un'imagine su la natura,
A la limite, le terme idea est impropre. Il porte à confusion, il oblige au contresens. Bellori, d'ailleurs, varie les termes. Parfois il emploie, au lieu d'Idée, le terme de modèle (essempio) ou le terme d'image (imagine), parfois celui de forme (forma). Dans la vignette destinée au Dominiquin il en vient au vieux terme de la critique littéraire concettoconceptus auquel il associe imaginatio-fantasia, tant il est incapable de définir l'idée indépendamment de sa génèse. Le personnage de la vignette, couronné des lauriers du poète, désigne de l'index à la fois son front et son oeil. Au fond, le Bernin, désignant à Chantelou son front pour décrire Poussin "comme un peintre qui travaille de là ", fut peut-être l'inspirateur de la vignette de Bellori ${ }^{77}$.

Dans sa biographie du Dominiquin, Bellori reprend les termes qu'il utilisait en 1664.

Il exprimait jusqu'à l'âme et à l'esprit [...]. Il contemplait sans cesse l'image des choses, retenant les formes des passions qui ne se font voir que par moments soudains et nc se peuvent autrement peindre d'après nature. Et voilà la difficulté principale de la peinture laquelle sans les mouvements des esprits n'est qu'imitation morte ${ }^{78}$.

Les deux allégories suivantes peuvent sembler étranges dans ce contexte (Figs 49 et 50 ). Toutes deux avaient été employées dans les allégories gravées de P. Testa, dans le Liceo (Fig. 52), dans l'Allégorie de la Peinture (de 1637 environ) (Fig. 62) et dans le Triomphe de la Peinture du début des années 1640 (Fig. 63) ${ }^{79}$. L'Allégorie de la Peinture montre le personnage principal couronné d'un palmier entier comme l'Hercule à la Croisée des chemins d'Annibale Carrache. A sa gauche une Diane d'Ephèse, symbole traditionnel de la généreuse abondance de la Nature. Derrière elle les trois Grâces. On retrouve les trois Grâces couronnant la Peinture dans le Triomphe. Dans un dessin préparatoire pour la partie centrale du Liceo, Testa avait pensé représenter le concept de Nature par la Diane d'Ephèse. Pourquoi la Nature est-elle associée au peintre parfait? Bellori a déjà répondu : le concept, le modèle imaginaire doit se former, s'entretenir, se perfectionner dans et par l'observation continue de la Nature, puisque c'est

osservando le commozioni umane ed accompagnando li moti del corpo con li moti dell'animo; in modo che gli uni da gli altri dipendino vicendevolmente" (Bellori, Vite, 20).

77 P. Fréart de Chantelou, Journal de voyage du Chevalier Bernin en France (1930), 110.

78 "Esprimeva sino all'anima ed alla mente [...] sempre contemplava l'imagine delle cose, ritenendo le forme de gli affetti, li quali non si veggono se non per subiti momenti, né possono in altuo modo ritrarsi dal naturale. F questa à la maggior difficoltà della pittura, la quale senza li movimenti dello spirito non è altro che una morta imitazione "(Bellori, Vite, 360)

79 Bartsch, Le peintre graveur, xx, 223 et 226. 
de la Nature que vient l'idé $e^{80}$. Les trois soeurs qui sont associées à l'Algarde sont quelque peu ambiguës (Fig. 50). Notons une fois de plus que l'association au personnage historique que fut l'Algarde n'offre aucun caractère de nécessité. Dans la vignette, les trois soeurs tiennent les attributs des trois arts du dessin, peinture, sculpture, architecture (?), et sont groupées en cercle comme les trois Grâces, et enlacées comme elles. Il semble assez clair que le concept formateur de cette image fut celui des trois Charites, associées à la peinture triomphante, concept auquel vint s'ajouter l'allusion aux trois arts par référence peut-être au Dominiquin qui était, nous dit Bellori, aussi bon architecte que peintre.

La vignette associée au Poussin est d'un intérêt tout à fait exceptionnel (Fig. 51). Lumen et Umbra : un personnage désigne une planche où l'on voit la représentation de solides. D'autres solides, cône, cylindre, cube sont situés derrière le personnage. Que signifie cette allégorie? Une apparente solution au problème est donnée par le portrait gravé de Poussin situé sur la page précédente (Fig. 64). Le peintre tient un livre dont on voit le titre "De. I.UM. et UMB. ", c'est-à-dire de Lumine et umbra, allusion au traité de théorie de la peinture que Poussin projetait d'écrire et dont Bellori a reproduit quelques fragments à la fin de la biographie du peintre, en fait des notes de lecture copiées par Poussin ${ }^{81}$. Cette gravure a été faite d'après l'autoportrait de 1650 du Poussin (Fig. 65). Sa fidélité au modèle est assez relative. L'autoportrait peint montre le peintre tenant le livre mais sans aucune inscription sur la tranche. Derrière lui, par contre, apparaît un personnage d'un tableau : une femme portant un diadème au centre duquel est figuré un oeil. L'étrangeté même de la représentation incite à en chercher la source dans une iconologie. On la trouve aisément chez Ripa: il s'agit de la perspective.

Une lemme très bellc et gracieuse; cllc portera au cou un collier d'or ayant $\mathrm{cn}$ pendentif un oeil humain [...]. Son vêtement, de couleur sombre vers le bas, s'éclaircit au fur et à mesure que l'on monte et vers la tête il est de coulcur très claire [...]. Ceci démontre que les opérations dc la perspective se font à l'aide de la clarté de la lumièrc et à l'aide de l'obscurité de l'ombre, avec une certaine gradation selon les distances et les rellets ${ }^{82}$.

80 $\Lambda$ cette image de la nature s'associe l'idée de sostanza décrite par Ripa, Iconologia (1603), 468, et qui presse ses mamelles pour en faire jaillir le lait.

81 Bellori, Vite, 478-81. C'est dans l'autoportrait de 1649, à Berlin, que Poussin tient un livre intitulé De lumine et colore.

82 " Ionna di bellissimo e gratioso aspetto; haverà al collo una collana d'oro c'habbia per pendente un'occhio humano [...] ncl vestimento da piedi sarà il color oscuro, e di mano in mano ascendendo sarà piu chiaro, tanto che da capo venga ad csser chiarissimo. . I i colori nelle vesti variati da oscuro
Bellori avait décrit ainsi l'autoportrait de 1650 :

Derrière lui sur l'autre tableau cst figurée la têtc d'une femme de profil, un oeil est figuré sur son diadème. C'est la Peinture ${ }^{83}$.

Ceci ne signifie pas que Bellori ignorait qu'il pût s'agir de la perspective. Bien au contraire. Cela signifie que pour lui peinture et perspective sont synonymes. Dans la gravure du portrait de Poussin chez Bellori, le personnage couronné n'apparaît pas. Par contre, la vignette joue le rôle qui lui était réservé dans le tableau. Il existe selon Ripa une seconde façon de représenter la perspective: "Une femme qui, de ses deux mains tienı une perspective; à ses pieds, des équerres, des compas et autres instruments de cet art ${ }^{84}$.

C'est bien ce que représente la vignette (Fig. 5̄l). Les instruments ont été remplacés par les solides, eux-mêmes représentés en perspective. Si Poussin s'est représenté lui-même accompagné de la perspective, la vignette trouve sa justification suffisante dans la biographie à laquelle elle est associée, tout comme la vignette du Conceptus-imaginatio s'appliquait au caractère particulier de l'art du Dominiquin. Bellori n'a pas laissé passer l'occasion de préciser comment Poussin fut perspectiviste:

Quand il voulait réaliser l'un de ses tableaux, après en avoir réglé la composition, il en dressait une esquisse [...] puis il façonnait de petits modìles de cire de toutes les figures dans leurs attitudes[...]. [II] Composait l'histoire ou la fable cn relief pour voir les effets naturels de la lumière et cles ombres des corps ${ }^{85}$.

Mais la présence de la perspective se justifie-telle à l'intérieur de la définition de la peinture

al chiaro sono per dimostrare che l'operationi della prospettiva si fanno col chiaro della luce, e con l'oscuro dell'ombra con una certa graduatione, secondo le distantie, e riflessi" (Ripa, Iconologia [160)3], 419). Pour l'interprétation de l'iconographie de l'autoportrait de Poussin on sc reportera à l'ouvrage de G. Kauffmann, Poussin-Studien (Berlin, 1960). L'allégorie de la perspective a été analyséc par D. Posner, "The Picture of Painting in Poussin's Selfportrait ". dans Essays in the History of Art Presented to Rudolf Wittkower (london, 1967), I1, 20()-203. Pour une réévaluation générale du sens de cet autoportrait on lira l'article de M. Winner, "Poussins Selbstbildnis im I.ourre als Kunsttheoretische Allegorie ", Römisches Jahrbuch für Kunstgeschichte, $\mathrm{xx}$ (1983), 418-49.

83 "Dietro nell'altra tavola contraria è figurata la testa di una donna in profilo con un occhio sopra la fronte nel diadema : questa è la Pittura "(Bellori. Vite, 455).

84 Ripa. Iconologia (1603), 419. Prospettiva, deuxième définition: " Domna, che con ambe le mani tiene una prospettiva, e alli piedi hà squadre, compassi, c altri stromenti convenevoli a quest'arte".

85 "Quando voleva fare i suoi componimenti, poichè aveva concepita l'invenzione, ne segnava uno schizzo quanto gli bastava per intenderla; dopo formava modeletti di cera di tutte le figure nelle loro attitudini. . e ne componeva l'istoria o la favola di rilievo, per vedere gli effetti naturali del lume e dell'ombre de'corpi "(Bellori, Vite, 4j2). 
idéale de Bellori? Oui, et c'est peut-être de tout le programme l'opération la plus étonnante. Si l'on compare la Geometria - en fait la Mathématique-du premier groupe à la géométrie dans l'espace du troisième groupe, il est facile de comprendre le projet de Bellori: la peinture idéale résout l'abstraction mathématique en représentation spatiale, dans la lumière, dans la zone solaire d'Aglaia. Mais cela aussi Bellori l'a, semble-t-il, emprunté à P. Testa. Au centre du Liceo vers la gauche (Fig. 52), au premier plan, Testa a figuré la peinture, jeune et gracieuse, conduite par l'optique vers le groupe des mathématiciens. L'optique tient une planche sur laquelle sont figurés des schémas et des figures, qui rappellent certains dessins d'optique et de perspective de Léonard (Fig. 66) ${ }^{86}$, l'optique c'est-à-dire la perspective, comme le précise Testa dans son libretto: " [...] l'optique pour la raison des plans, des lumières, des ombres et tout ce qui apparaît à l'oeil par la raison d'un point $[\ldots] »{ }^{87}$.

C'est par l'optique, en effet, que la mesure de la terre par la Praxis et les raisons spéculatives de la Mathématique s'accordent pour faire naître la Peinture.

86 Codex Urbinas Latinus, 1270, in fac-similé, fol. 213r.

87 " [...] l'otica per la ragione dei piani, dei lumi, de[l]le onbre el tutto ciò che hapare al ochio per la ragione d'un pun[to] " (Cropper, The Ideal of Painting. 228). 